SPENCER CHENG

\title{
Tratamento dos tumores avançados da junção esofagogástrica por prótese auto-expansível com
} válvula anti-refluxo

Dissertação apresentada à Faculdade de Medicina da Universidade de São Paulo para obtenção do Título de Mestre em Ciências Área de concentração: Cirurgia do Aparelho Digestivo

Orientador: Dr. Eduardo Guimarães Hourneaux de Moura

São Paulo 2005 
Aos meus pais, Simon e Elizabeth, exemplos de coragem e perseverança, pelo constante amor, compreensão e orientação.

À minha avó Lucy, semente de retidão responsável pelos frutos da nossa família.

Aos meus irmãos, Henrique e Isabela, pela eterna amizade e companheirismo. 
Agradecimentos

Ao Dr. Shinichi Ishioka, fonte de sabedoria e honestidade, por quem guardo minha eterna admiração.

Ao Prof. Dr. Paulo Sakai, cientista obstinado, pelo estímulo incessante à pesquisa.

Ao Dr. Eduardo Guimarães Hourneaux de Moura, orientador desta dissertação, pelo exemplo de perseverança, amizade e dedicação.

Ao Prof. Dr Joaquim Gama Rodrigues, égide da Pós-graduação da Cirurgia do Aparelho Digestivo do HCFMUSP.

Ao Prof. Dr. Ivan Cecconello pelos ensinamentos e apoio recebidos para a realização deste estudo.

Ao Prof. Dr. Valter Nilton Félix, pelas imprescindíveis sugestões à esta dissertação.

À Dra. Jeane Martins Melo, pelo suporte incessante em todos os momentos desta dissertação.

Ao Dr. Sérgio Eiji Matuguma, pela grandeza da sua amizade em todos os momentos. 
Aos amigos do Serviço de Endoscopia Gastrointestinal do HCFMUSP: Dr. Sebastião Alves D’Antônio, Dr. Koshiti Hondo, Dr. Hélio Batista, Dr. Marcos C. Visconti, Dr. José Robledo Naves, Dr. Toshiro Tomishigue, Prof. Dr. Fauze Maluf Filho, Dr. Dalton Marques Chaves, Dr. Renato Baracat, Dra. Eunice Komo Chiba, Dra. Sônia Nádia Fylyk, Dr. Edson Ide, Dra. Adriana Vaz Safatle-Ribeiro, Dra. Elisa Ryoka Baba, Dr. Rogério Kuga, Dr. Everson Artifon, Dr. Sérgio Shigetoshi Ueda, Dr. Nelson Tomio Miyajima, Dr. Gilson Kamiyama, Dr. Fábio Hondo, Dr. Carlos Kiyoshi Furuya Jr, Dr. Robson Kiyoshi Ishida, Dr. Eduardo Grecco e aos médicos estagiários dos anos de 2004 e 2005.

A todos os funcionários da enfermagem e secretaria do Serviço de Endoscopia Gastrointestinal e Instituto de Radiologia do HCFMUSP, em especial a Sra. Maria José Rosa.

Aos amigos Dr. Fabrício da Silveira Bossi, Dr. Orestes Previtale Júnior, pela rica convivência diária.

Aos meus professores da arte médica: Dr. Admar Concon Filho, Dr. Nelson Simões, Dr. João Moysés Abujadi, Dr. Ruy Meirelles dos Santos, Dr. Marco Antônio de Campos Moreira, Dr. Moacir Valente de Oliveira, Dr. Carlos Hermenegildo Bissotto, Dr. Carlos D’Ancona, Dr. Adilson Gouveia, Dr. Paulo Sérgio Ribeiro, Dr. Vicente Nacarato, Dr. Sidney Macedo, Dr. Eduardo Barel, Dr. Erlon José Geraldo, Dra. Maria Fernanda Costa Haddad, Dr. Manoel Joaquim Eiras Falcão, Dra. Desanka Dragosavac, Dr. Antônio Falcão, Dr. Abdel Latif e aos médicos anestesiologistas Dr. Roberto, Dr. Décio, Dr. Edson, Dr. Milton, Dr. Luis Fernando, Dr. Mário. 
Aos médicos residentes da Irmandade Santa Casa de Misericórdia de Valinhos, pelo estímulo constante ao aprendizado. 


\section{Resumo}

Cheng S. Tratamento dos tumores da junção esofagogástrica por prótese autoexpansível com válvula anti-refluxo. 2005. Dissertação (Mestrado)- Faculdade de Medicina, Universidade de São Paulo, São Paulo, 2005.

A paliação da disfagia por neoplasia maligna avançada da junção esofagogástrica com próteses metálicas auto-expansíveis propicia condições para o refluxo gastroesofágico e complicações como broncoaspiração e esofagite. Foram desenvolvidas próteses com mecanismo anti-refluxo para evitá-las. No presente estudo, os resultados clínicos obtidos com o tratamento paliativo em 30 pacientes com neoplasia inoperável de esôfago distal e/ou cárdia pela colocação da prótese metálica autoexpansível do esôfago com válvula anti-refluxo (Esophageal Z-stent W/DUA Antireflux valve - Wilson-Cook®) foram avaliados no período de 2001 a 2004. Sintomas de disfagia, broncoaspiração, pirose, regurgitação e dor retroesternal foram registrados previamente à passagem da prótese e após sete, 30 e 120 dias. O funcionamento da prótese foi avaliado por esofagograma baritado e endoscopia digestiva alta, realizados respectivamente sete e 30 dias após o procedimento. O parâmetro nutricional utilizado foi o índice de massa corpórea (IMC) e a qualidade de vida foi verificada pelo índice Karnofsky, previamente e após 30 dias. As complicações imediatas e tardias foram registradas. Não houve alteração nos resultados de índice de massa corpórea $(p=0,104)$ e qualidade de vida $(p=1.00)$. Houve melhora da disfagia nas avaliações do sétimo, $30^{\circ} \mathrm{e}$ $120^{\circ}$ dias $(p<0,001 ; p<0,001$ e $p=0,010$ respectivamente), assim como a regurgitação $(p=0,001 ; p=0,008$ e $p=0,039$ respectivamente). Após a colocação da prótese, observouse aumento da dor retroesternal no sétimo, $30^{\circ}$ e $120^{\circ}$ dias de avaliação $(p=0,011$; $p=0,025$ e $p=0,020$ respectivamente), sem piora da pirose $(p=0,916 ; p=0,786 ; p=0,655)$, 
eructação $(p=0,417 ; p=0,257 ; p=0,655)$ e tosse $(p=0,410 ; p=0,602 ; p=0,888)$. A complicação imediata documentada foi perfuração (10\%), e as tardias foram hemorragia $(23,3 \%)$, ruptura da válvula $(20 \%)$, hiperplasia tecidual benigna $(13,3 \%)$ e migração (13,3\%). A prótese auto-expansível com válvula anti-refluxo foi eficaz na paliação da disfagia, impediu ocorrências de manifestações de broncoaspiração, não interferiu na evolução do parâmetro nutricional e tampouco na avaliação da qualidade de vida. 


\section{Abstract}

Cheng S. Antireflux valve self-expanding stent in the treatment of gastroesophageal junction tumors. 2005. Thesis (Master)- Faculdade de Medicina, Universidade de São Paulo, São Paulo, 2005.

Advanced tumors of distal esophagus and cardia have been treated with self-expanding metal stents deployed across gastroesophageal junction (GEJ). This creates conditions for gastroesophageal reflux and complications like aspiration and esophagitis. Self-expanding metal stent with antireflux mechanism was developed to avoid these complications. Thirty patients with cancer at the GEJ were studied in palliative therapy with Esophageal Z-stent W/DUA Antireflux valve (Wilson Cook ${ }^{\mathrm{TM}}$ ) from 2001 to 2004. Dysphagia, aspiration, heartburn, regurgitation and chest pain symptoms were registrated before and after seven, 30 and 120 days the procedure. Quality of life by the Karnofsky index and nutritional parameter by Body mass index (BMI) were obtained previously and 30 days after. Stent performance was verified with barium study and upper GI endoscopy performed one week and one month after stent placement, respectively. Early and late complications were also registrated. There were no changes in body mass index or quality of life. Dysphagia improved seven, 30 and 120 days after stent placement $(p<0,001 ; p<0,001$ and $p=0,010$, respectively), same did regurgitation symptom $(p=0,001 ; p=0,008$ and $p=0,039)$. Chest pain increased in all evaluations $(p=0,011 ; p=0,025$ and $p=0,020)$ and there were no increase in heartburn $(p=0,916 ; p=0,786 ; p=0,655)$, belching $(p=0,417 ; p=0,257 ; p=0,655)$ or cough symptom $(p=0,410 ; p=0,602 ; p=0,888)$. Early complication was perforation (10\%). Late complications were bleeding (23,3\%), valve rupture $(20 \%)$, reactive tissue growth $(13,3 \%)$, and migration (13,3\%). Antireflux valve self-expanding metal stents were 
effective in dysphagia palliation and avoided clinical manifestations of aspiration and pneumonia. There was no influence in the nutritional parameter or quality of life index. 


\section{Lista de Abreviaturas}

$\begin{array}{ll}\% & \text { - Porcentagem } \\ * & \text { - Valor considerado significativo } \\ < & \text { - Menor } \\ = & \text { - Igual } \\ > & \text { - Maior } \\ \pm & \text { - Mais ou menos } \\ \text { ADC } & \text { - Adenocarcinoma } \\ \text { CEC } & \text { - Carcinoma Espinocelular } \\ \text { dp } & \text { - Desvio-padrão (variável quantitativa) } \\ \text { F } & \text { - French } \\ \text { h } & \text { - Hora } \\ \text { HCFMUSP } & \text { - Hospital das Clínicas da Faculdade de Medicina da } \\ & \text { Universidade de São Paulo } \\ \text { Ikc } & \text { - Condicaão global } \\ \text { Ikp } & \text { - Aptidão física } \\ \text { IMC } & \text { - Índice de massa corpórea } \\ \text { INCA } & \text { - Instituto Nacional do Câncer } \\ \text { JEG } & \text { - Junção esofagogástrica } \\ \text { kg } & \text { - Kilograma } \\ \text { Laser } & \text { - Light Amplification by Stimulated Emisson of Radiation } \\ \text { m² } & \text { - Metro quadrado } \\ \text { mg } & \text { - Miligrama } \\ \text { ml } & \text { - Mililitro } \\ \text { n } & \text { - Variável qualitativa de freqüência absoluta } \\ \text { N/mm/cm } & \text { - Newton/milímetro/centímetro } \\ \text { Nd-YAG } & \text { - Neodymium- Yttrium Aluminum Garnet } \\ \text { oC } & \text { - Grau Centrígrado } \\ \text { p } & \text { - Nível descritvos } \\ \text { PA } & \text { - Póstero-anterior } \\ \text { pH } & \text { - Potencial de hidrogênio } \\ \text { PMAE } & \text { - Prótese metálica auto-expansível } \\ \text { QT } & \text { - Quimioterapia } \\ \text { RGE } & \text { - Refluxo gastroesofágico } \\ \text { RT } & \text { - Radioterapia } \\ \text { TNM } & \text { - Estadiamento de tumores baseado nos critérios Tumor, } \\ & \text { Linfonodo, Metástase. } \\ \text { UICC } & \text { - União Internacional Contra o Cãncer } \\ \text { VAR } & \text { - Válvula anti-refluxo } \\ \alpha & \text { - nível de significância } \\ & \end{array}$




\section{RESUMO}

\section{LISTA DE ABREVIATURAS}

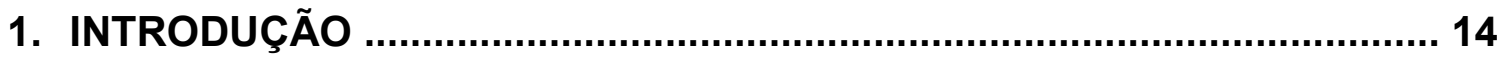

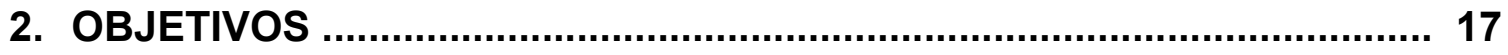

3. CASUÍSTICA E MÉTODOS …............................................................ 19

a. CRITÉRIOS DE SELEÇÃO DE PACIENTES .................................. 19

b. ADMISSÃO PARA O ESTUDO .................................................... 20

c. PROTOCOLO DE AVALIAÇÃO …………………............................ 20

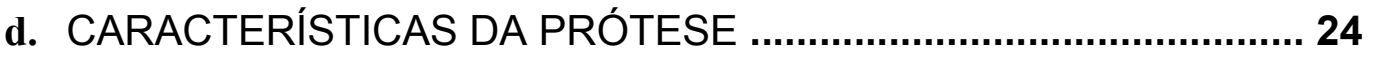

e. PROCEDIMENTO DE COLOCAÇÃO DA PMAE .............................. 25

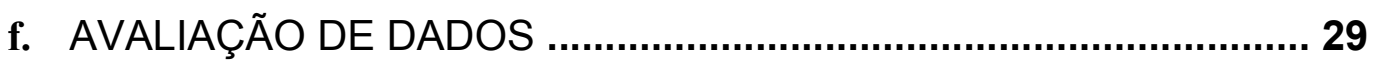

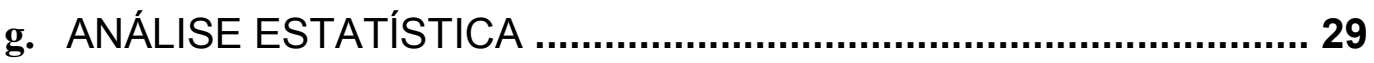

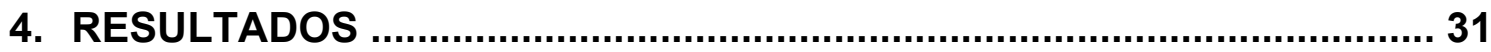

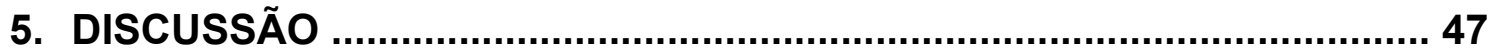

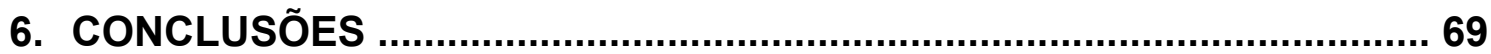

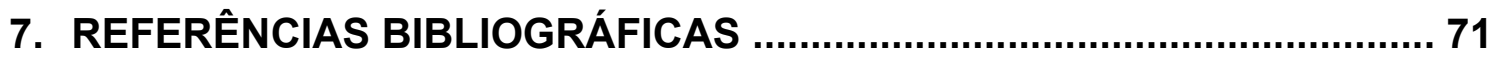


Introdução 


\section{Introdução}

O câncer de esôfago apresenta prognóstico desfavorável quando se iniciam os primeiros sintomas. Metade dos pacientes apresenta tumor irressecável no momento do diagnóstico, e as taxas de sobrevida em cinco anos são de 5-10\% (BARON TH, 1999; DUA KS, 2001; ENZINGER PC, 2003; LUI JF, 2004; SIERSEMA PD, 2003).

Em 1990, essa enfermidade representava a sétima neoplasia maligna mais freqüente no mundo (PARKIN, 1999) e, no Brasil, dados do Instituto Nacional do Câncer (INCA) de 2000 indicaram como a sexta causa de mortalidade por câncer.

O tratamento para a neoplasia de esôfago, nas últimas décadas, recebeu novas perspectivas, com o avanço das técnicas cirúrgicas, anestesiológicas e diagnósticas. A abordagem dos pacientes atualmente é mais individualizada, com destaque ao estadiamento preciso da doença e à seleção dos pacientes passíveis de tratamento curativo, representado pelas técnicas cirúrgicas de ressecção (LAW S, 2004; TYTGAT, 2004).

No entanto, mais de $70 \%$ dos casos diagnosticados apresentam neoplasia avançada com estádio III e IV da classificação TNM (SALLUM RA, 1996). Para esse grupo de pacientes sem possibilidades de cura, há uma diversidade de opções paliativas, tais como cirurgias de derivação, radioterapia, quimioterapia e métodos endoscópicos.

O emprego de próteses metálicas auto-expansíveis (PMAE) representa forma segura e eficaz no tratamento paliativo da disfagia por malignidade. Nas últimas décadas, observou-se aumento na incidência de adenocarcinoma de esôfago distal e cárdia nos países ocidentais (BLOT WJ, 1991; VISCAINO AP, 2002). Conseqüentemente, pacientes com tumores irressecáveis começaram a ser tratados com PMAE através da junção 
esofagogástrica (JEG). No entanto, o seu emprego é contestado no tratamento dos tumores dessa região, porque está relacionada com alto índice de migração e estabelece comunicação permanente entre as duas cavidades, possibilitando refluxo gastroesofágico (RGE), regurgitação, pneumonia aspirativa, com impacto na qualidade de vida e sobrevida (BARTELSMAN JFW, 2000; DO YS, 2001; EL-SERAG HB, 2002; LAASCH HU, 2002; SONG, HY 1994).

O avanço tecnológico na elaboração de dispositivos mais seguros e eficazes permitiu o surgimento de próteses com mecanismo anti-refluxo, vislumbrando-se a possibilidade de evitar essas complicações.

Dua et al. (2002), desenvolveram PMAE com válvula anti-refluxo (VAR), que foi testada in vitro e in vivo, com resultados promissores na prevenção do refluxo e na paliação da disfagia. 
Objetivos 


\section{Objetivos:}

Avaliação da paliação da disfagia, das manifestações broncoaspirativas, de parâmetro nutricional e da qualidade de vida no tratamento paliativo de pacientes com tumores avançados da junção esofagogástrica, mediante o emprego da prótese metálica auto-expansível com válvula anti-refluxo. 
Casuística e métodos 


\section{Casuística e Métodos:}

No período de 2001 a 2004, 32 pacientes com neoplasia avançada da junção esofagogástrica foram tratados paliativamente por PMAE com VAR e acompanhados no Serviço de Endoscopia Gastrointestinal do HC-FMUSP.

As próteses foram inseridas pelo autor e o orientador do estudo, e o acompanhamento e avaliação clínica dos pacientes foram realizados somente pelo autor.

\section{Critério de seleção de pacientes:}

a) População de base

A população de base foi definida como pacientes apresentando disfagia decorrente de carcinoma inoperável de esôfago distal ou cárdia. A avaliação dos pacientes e a definição de tumores irressecáveis ou inoperáveis foi efetuada no Serviço de Cirurgia do Esôfago do HCFMUSP, que encaminhou tais pacientes ao Serviço de Endoscopia Gastrointestinal do HCFMUSP, para colocação da prótese.

b) Critérios de inclusão

- Pacientes com estadiamento TNM (UICC) Estádio III//V ${ }^{8}$, com obstrução maligna inoperável do esôfago distal e/ou cárdia.

c) Critérios de exclusão

- Pacientes com fístula esofagotraqueal ou esofagobrônquica, ou ambos.

- Pacientes com lesão neoplásica esofágica com extensão superior a 12 $\mathrm{cm}$. 
- Pacientes submetidos a procedimento cirúrgico do esôfago e/ou do estômago.

\section{Admissão para o estudo:}

Os pacientes envolvidos no estudo preencheram todos os critérios de seleção, foram informados sobre seus objetivos, estrutura, riscos e assinaram o formulário de consentimento informado.

\section{Protocolo de avaliação:}

A avaliação foi realizada por visitas dos pacientes ao Serviço de Endoscopia Digestiva do HCFMUSP, mediante o seguinte cronograma: avaliação inicial (prévia à colocação da prótese) e após sete, 30 e 120 dias. Os pacientes foram acompanhados até o óbito ou término do estudo. O protocolo consistia nas seguintes informações:

1. A identificação do paciente com nome, sexo, idade, peso, índice de massa corpórea e índice de Karnofsky.

O índice de massa corpórea foi calculado de acordo com a fórmula:

$$
\operatorname{IMC}\left(\mathrm{kg} / \mathrm{m}^{2}\right)=\frac{\text { peso atual }(\mathrm{kg})}{\text { altura }(\mathrm{m})^{2}}
$$

Os valores considerados como normais para ambos os sexos variam de 18,5 a $27 \mathrm{~kg} / \mathrm{m}^{2}$. 
O índice de Karnofsky (1949), tendo como base a condição global (Ikc) e a aptidão física (lkp), foi obtido previamente ao procedimento e 30 dias após a colocação da prótese, com o seguinte questionário:

- Condição global:

A- capaz de manter atividade normal e trabalhar. Nenhum cuidado especial é necessário.

B- Incapaz de trabalhar. Capaz de viver em casa e manter seus cuidados pessoais mais comuns. Um grau variado de ajuda é necessário.

C- Incapaz de cuidar de si próprio. Requer ajuda equivalente a cuidados institucionais ou hospitalares. A doença pode estar evoluindo rapidamente.

- Aptidão física:

100\% normal. Sem queixas. Sem evidências de doenças;

90\% capaz de exercer as atividades normais. Sinais e sintomas menores da doença;

$80 \%$ atividade normal com esforços. Alguns sinais e sintomas da doença;

$70 \%$ capaz de cuidar de si próprio. Incapaz de levar atividade normal ou trabalhar ativamente;

$60 \%$ requer assistência ocasional, mas é capaz de realizar a maior parte das suas necessidades pessoais;

$50 \%$ requer assistência considerável e cuidado médico freqüente;

40\% incapaz. Requer cuidados especiais de assistência;

$30 \%$ gravemente incapaz. Hospitalização está indicada embora a morte não seja iminente; 
$20 \%$ hospitalização necessária com importante necessidade de tratamento de apoio. Doença ativa;

$10 \%$ moribundo. Processo fatal em rápida progressão;

$0 \%$ morto.

2. A capacidade do paciente para ingerir e/ou deglutir alimentos foi classificada utilizando a escala de Atkinson e Ferguson (MOSCA F, 2003):

\begin{tabular}{|l|l|}
\hline Grau & Definição \\
\hline 0 & Deglutição normal \\
\hline 1 & Habilidade para ingerir alguns sólidos \\
\hline 2 & Habilidade para ingerir semi-sólidos \\
\hline 3 & Habilidade para ingerir somente líquidos \\
\hline 4 & Disfagia absoluta \\
\hline
\end{tabular}

3. O sintoma de dor retroesternal foi graduado por uma escala analógica visual de 0 a 10 assinalada pelo paciente:

Sem dor

Dor intensa

\begin{tabular}{|l|l|l|l|l|l|l|l|l|l|l|}
\hline 0 & 1 & 2 & 3 & 4 & 5 & 6 & 7 & 8 & 9 & 10 \\
\hline & & & & & & & & & & \\
\hline
\end{tabular}

4. Os sintomas relacionados ao RGE como pirose retroesternal, regurgitação, tosse, capacidade de eructação, também foram graduados em uma escala analógica visual de 0 a 10, conforme a intensidade: 


\begin{tabular}{|c|l|l|l|l|l|l|l|l|l|l|l|}
\hline & 0 & 1 & 2 & 3 & 4 & 5 & 6 & 7 & 8 & 9 & 10 \\
\hline Pirose & & & & & & & & & & & \\
\hline Regurgitação & & & & & & & & & & & \\
\hline Tosse & & & & & & & & & & & \\
\hline Eructação & & & & & & & & & & & \\
\hline
\end{tabular}

5. Complicações como perfuração, sangramento, impactação de bolo alimentar e migração da prótese foram registrados.

6. Exames complementares realizados:

- Pré-procedimento : Radiografia simples de tórax

Tomografia computadorizada de tórax e abdome

Ultra-sonografia de abdome superior

Endoscopia digestiva alta

- Pós-procedimento:

Radiografia simples de tórax
Radiografia contrastada de esôfago e estômago
Endoscopia digestiva alta

\section{Exames radiológicos}

- Radiografia simples de tórax póstero-anterior (PA) e perfil: para estadiamento de metástases pulmonares, diagnóstico de pneumonia e complicações.

- Radiografia contrastada do esôfago e estômago: realizada sete dias após a colocação da prótese, para verificar a permeabilidade da mesma e o funcionamento da VAR, com o 
paciente em posição ortostática, decúbito dorsal horizontal e em posição de Trendelemburg.

- Tomografia computadorizada de Tórax e Abdome: para estadiamento da neoplasia (aderência com a aorta, abaulamento e invasão de traquéia e broncofonte, corrosão de corpos vertebrais, metástases ganglionares, pulmonares e hepáticas).

- Ultra-sonografia de abdome: para estadiamento da neoplasia.

Endoscopia digestiva alta: realizada antes do procedimento, para determinar a localização da lesão, extensão, acometimento circunferencial e características da neoplasia, além da obtenção de biópsias para histologia; outro exame foi realizado no $30^{\circ}$ dia e no surgimento de sintomas relacionados ao RGE, para verificar o posicionamento da prótese, sua permeabilidade e funcionamento da válvula.

\section{Características da prótese}

O modelo de prótese utilizado nesse estudo é a Esophageal Z-Stent W/DUA Antireflux Valve, Wilson-Cook® (DUA Z-Stent). A prótese possui estrutura metálica de aço inoxidável disposta em "zigue-zague", formando "gaiolas" de $2 \mathrm{~cm}$ cada, revestida por poliuretano em toda a extensão, com exceção da extremidade proximal. O diâmetro interno da porção central mede $18 \mathrm{~mm}$ e os limites proximal e distal $25 \mathrm{~mm}$. A força de expansibilidade é igual a $0.283 \mathrm{~N} / \mathrm{mm} / \mathrm{cm}$. Os comprimentos disponíveis comercialmente são oito, 10, 12 e $14 \mathrm{~cm}$. A válvula anti-refluxo é prolongamento do revestimento de poliuretano na sua porção distal, estendendo-se por $80 \mathrm{~mm}$ além da parte metálica, com forma cilíndrica e espessura de $0.17 \mathrm{~mm}$ (Figura 1). 
O dispositivo de colocação (sistema introdutor) é composto por dois tubos plásticos, um externo, e outro interno radiopaco (empurrador), e apresenta na sua extremidade distal a ogiva introdutora, também radiopaca. Possui trava de segurança que impede o deslocamento do tubo interno, localizada proximalmente, e fio de segurança ligado ao limite superior da prótese. O sistema é atravessado longitudinalmente por canal para passagem de fio guia, seu comprimento é de $70 \mathrm{~cm}$ e diâmetro externo de $10 \mathrm{~mm}$ (Figura 2 e 3).

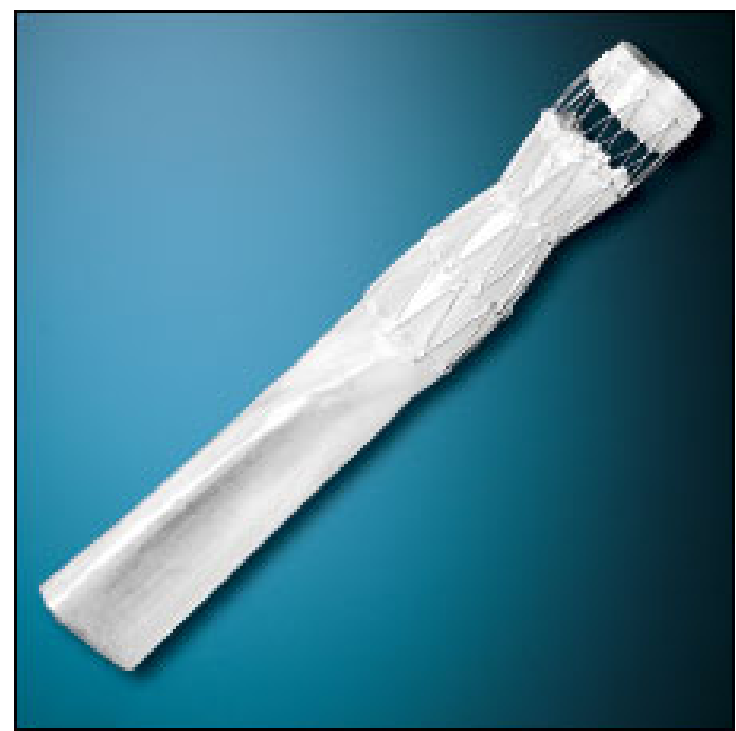

Figura 1- DUA Z-Stent

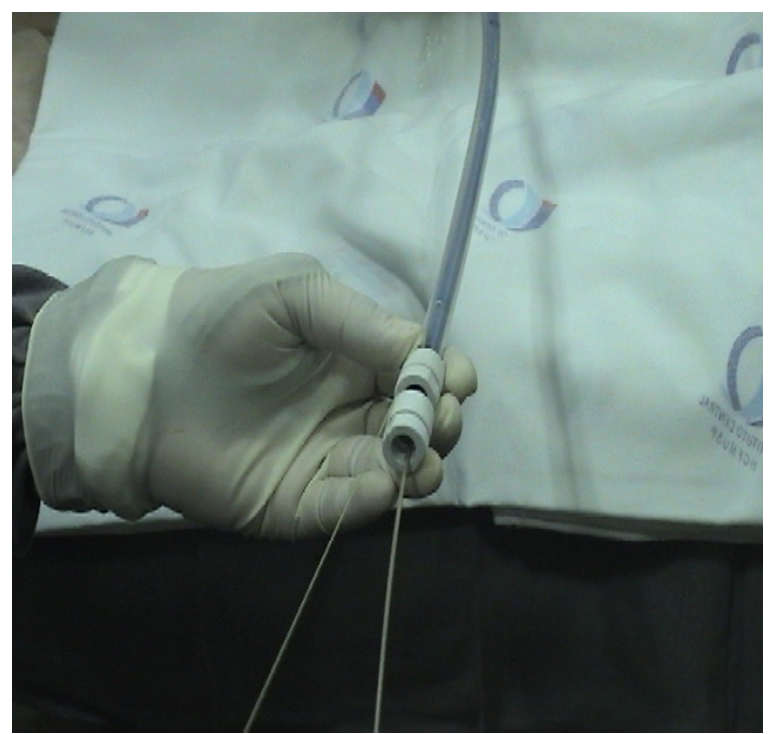

Figura 2- Porção proximal do sistema introdutor

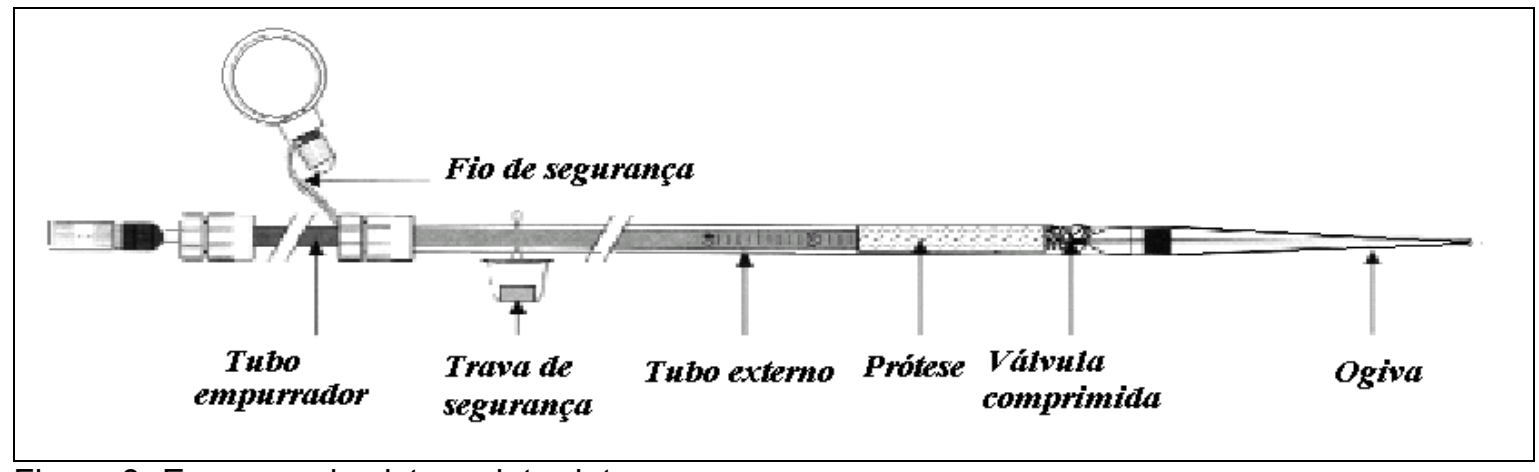

Figura 3- Esquema do sistema introdutor 


\section{Procedimento de colocação da PMAE}

Todos os pacientes foram submetidos a sedação-consciente durante a inserção da prótese. Os limites superior e inferior da neoplasia foram marcados com injeção de material contrastado radiopaco (Lipiodolß) (figura 4). A prótese foi posicionada dentro do esôfago com auxílio de fio guia, respeitando o procedimento de inserção descrito pelo fabricante. O posicionamento adequado foi realizado sob controle fluoroscópico. As extremidades da prótese expandida estenderam-se acima e abaixo dos limites proximal e distal da neoplasia por pelo menos 1-2 cm, respectivamente (figura 5).

Após ser lubrificada, a prótese e sua válvula são tracionadas inteiramente para dentro do tubo externo, por tração do fio preso à extremidade superior da prótese (figura $6 a, 6 b$ e $6 c)$. Com a ogiva posicionada e presa à extremidade inferior do tubo externo, a prótese é empurrada para baixo, para comprimir a válvula dentro do tubo externo. Em seguida, a gaiola metálica inferior é posicionada através da JEG, sem avançar excessivamente o sistema para dentro do estômago. Após posicionar a prótese comprimida através da lesão, o tubo empurrador é mantido em sua posição e o tubo externo retirado, liberando a válvula e permitindo a expansão da prótese.

Nos pacientes com estenoses apertadas, foram realizadas dilatações com sondas termoplásticas até o calibre de $11 \mathrm{~mm}$ (33 F). A dilatação para diâmetros maiores foi evitada, pelo risco de perfuração e por predispor à migração da prótese. Para prevenir que a extremidade distal da prótese ficasse encostada na parede gástrica, uma ou duas gaiolas metálicas $(2-4 \mathrm{~cm})$ foram projetadas para dentro do estômago. Contudo, para pacientes com hérnia hiatal, próteses mais longas foram utilizadas para unir o tumor à hérnia, evitando assim o aprisionamento da válvula dentro da mesma. 


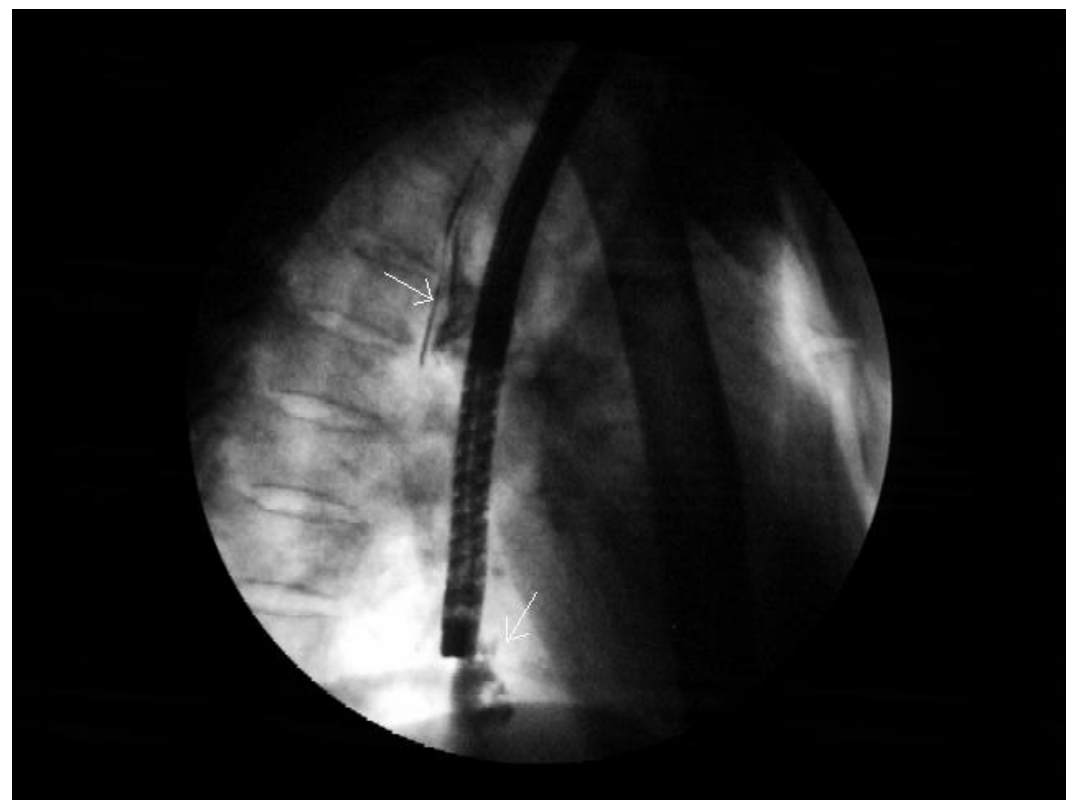

Figura 4- Marcação de limites proximal e distal (setas)

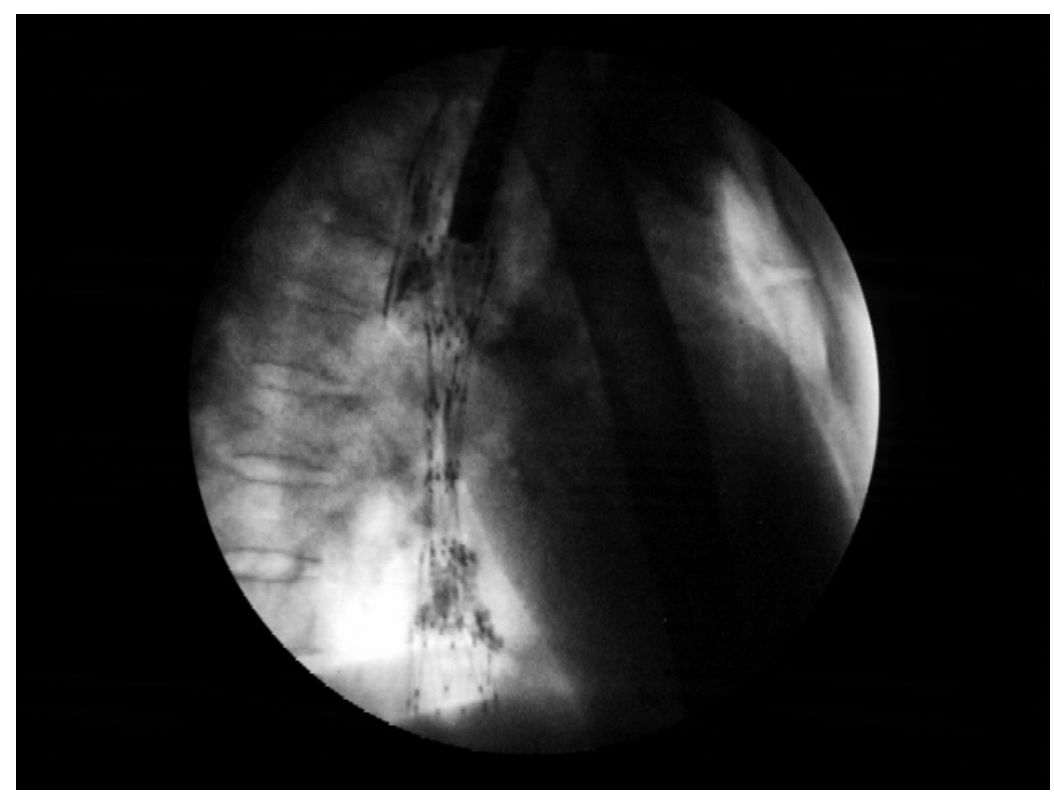

Figura 5- Prótese ultrapassando os limites radiopacos 


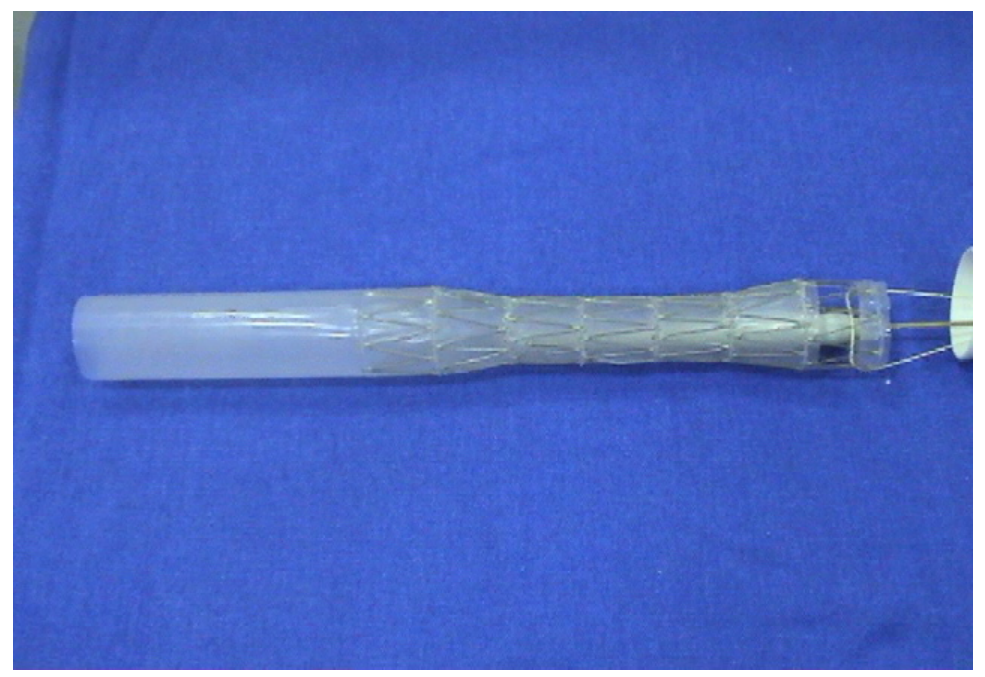

Figura 6a- Prótese antes da montagem

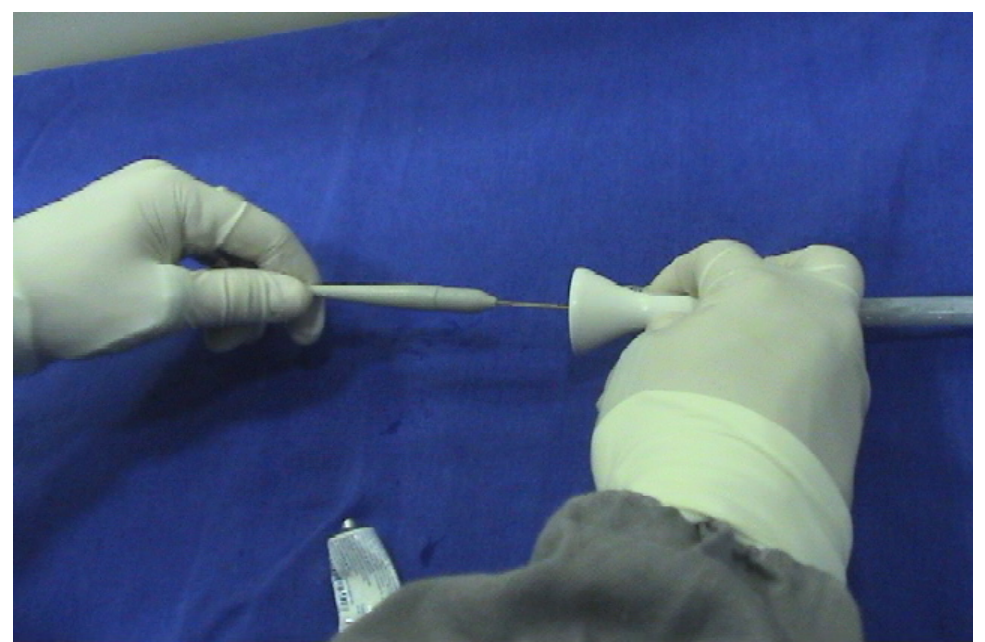

Figura 6b- Montagem da prótese no sistema introdutor.

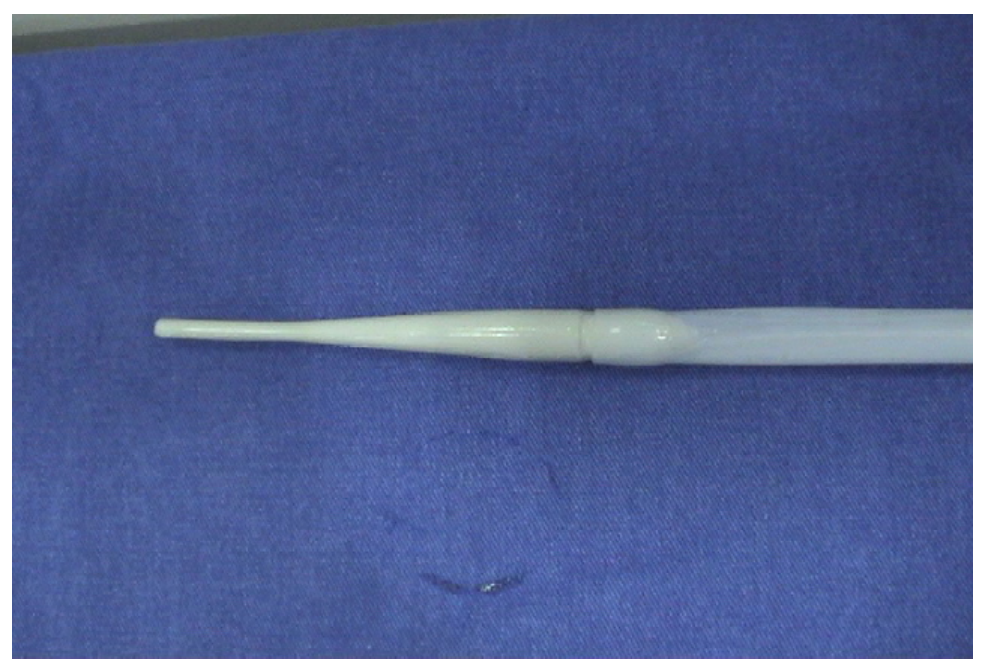

Figura 6c- Sistema introdutor montado. 


\section{Avaliação de dados:}

- Características básicas: idade e sexo;

- Características clínicas: índice de massa corpórea, índice de Karnofsky, grau de disfagia, dor retroesternal, pirose, regurgitação, eructação, tosse, terapia prévia com radioterapia ou citostáticos;

- Resultados e sobrevida;

- Complicações: perfuração, hemorragia, aspiração, migração da prótese;

\section{Análise estatística}

As variáveis quantitativas foram representadas por média e desvio-padrão (dp) e as qualitativas, por freqüência absoluta (n) e relativa (\%). A comparação entre os grupos de interesse quanto às variáveis qualitativas foi realizada pelo Teste Exato de Fisher. As curvas de sobrevida foram estimadas pelo método de Kaplan-Meier e os grupos de interesse comparados pelo teste Log-rank. A comparação entre o grau de sintomas nas avaliações pré-prótese e pós-prótese $\left(7^{\circ}, 30^{\circ}\right.$ e $120^{\circ}$ dias $)$ foi realizada pela Prova de Wilcoxon para amostras relacionadas.

Adotou-se o nível de significância de 0,05 ( $\alpha=5 \%$; níveis descritivos (p) inferiores a esse valor foram considerados significantes e representados por *.

Para avaliar se os pacientes sofreram alteração quanto ao IMC, foi realizada uma análise de variâncias com medidas repetidas com correção de Huyng-Feldt (NETER et al., 1996). 
Resultados 


\section{Resultados}

\subsection{Características dos pacientes}

Foram estudados 32 pacientes e dois desses excluídos, um por perda do acompanhamento e outro por desistência. Dos 30 pacientes com diagnóstico de neoplasia irressecável de esôfago e/ou cárdia, 25 (83,3\%) eram homens. A idade dos pacientes variou de 44 a 88 anos (média $=66,6$ anos; desvio-padrão $=12,5$ anos). Houve tendência de os pacientes do sexo masculino serem mais jovens (média de idade $=64,8$ anos; desvio-padrão $=11,9$ anos) que as pacientes de sexo feminino (média de idade $=$ 75,2 anos; 12,8 anos) $(p=0,09)$. Dezenove $(63,3 \%)$ pacientes apresentavam adenocarcinoma e $11(36,6 \%)$ carcinoma epidermóide. Não foi observada associação significativa entre o sexo e o tipo de tumor $(p=0,62)$.

\subsection{Sintomas presentes antes da colocação da prótese}

\begin{tabular}{lll}
\hline & N & $\mathbf{\%}$ \\
\hline Deglutição normal & 0 & 0 \\
Habilidade para ingerir alguns sólidos & 6 & 20,0 \\
Habilidade para ingerir semi-sólidos & 10 & 33,3 \\
Habilidade para ingerir somente líquidos & 5 & 16,7 \\
Disfagia absoluta & 9 & 30,0 \\
\hline
\end{tabular}




\subsection{Análises Estatísticas Inferenciais}

Gráfico 1.3.1 Valores médios de IMC e respectivos erros padrão para os

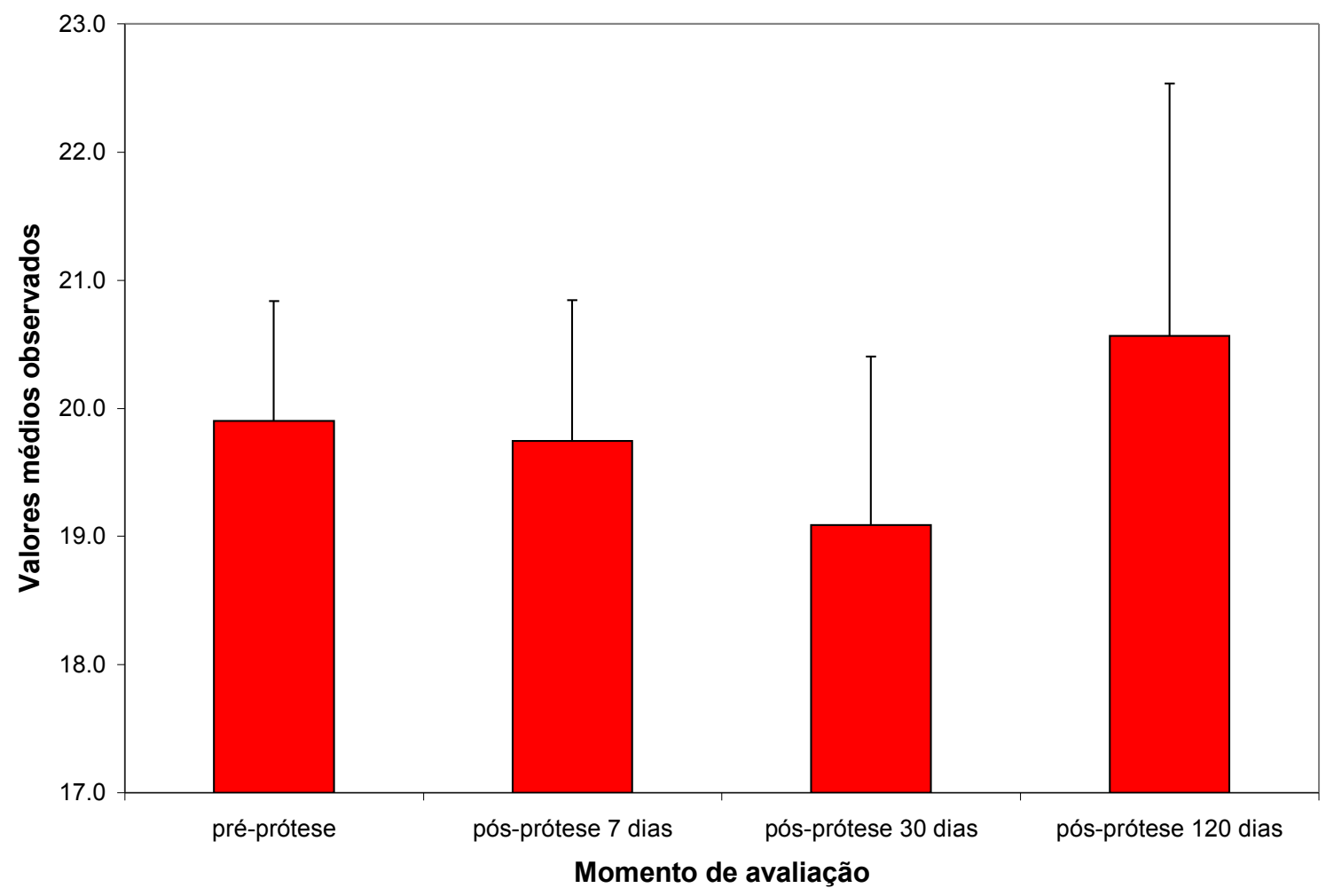

momentos de avaliação.

Pelo Gráfico 1.3.1, não há alteração média no IMC dos pacientes.

Tabela 1.3.1 Resultado da análise de variâncias com medidas repetidas para o IMC.

\begin{tabular}{lcccc}
\hline Fator & $\begin{array}{c}\text { Quadrado } \\
\text { Médio }\end{array}$ & gl & Valor F & P-value \\
\hline Momento & 8.15 & 1.54 & 2.87 & 0.104 \\
Erros & 2.85 & 12.34 & & \\
\hline
\end{tabular}

Pela Tabela 1.3.1, observa-se que em média não há alteração estatisticamente significativa do IMC $(p=0,104)$. 
Tabela 1.3.2 Avaliação do grau de disfagia.

\begin{tabular}{|c|c|c|c|c|c|}
\hline $\begin{array}{l}\text { Avaliação de } \\
\text { disfagia }\end{array}$ & grau pós & $\mathrm{n} \quad(\%)$ & & média $\pm d p$ & $p$ \\
\hline \multirow{4}{*}{$\begin{array}{l}7^{\circ} \text { dia } \\
\text { Pós-prótese }\end{array}$} & $<$ pré & $26 \quad(92,9)$ & \multirow{2}{*}{ pré-prótese } & \multirow{2}{*}{$2,59 \pm 1,15$} & \multirow{4}{*}{$<0,001^{*}$} \\
\hline & $=$ pré & $2(7,1)$ & & & \\
\hline & $>$ pré & $0(0,0)$ & \multirow{2}{*}{ pós-prótese } & \multirow{2}{*}{$0,69 \pm 0,85$} & \\
\hline & Total & $28 \quad(100,0)$ & & & \\
\hline \multirow{4}{*}{$\begin{array}{l}30^{\circ} \text { dia } \\
\text { Pós-prótese }\end{array}$} & $<$ pré & $19(82,6)$ & \multirow{2}{*}{ pré-prótese } & \multirow{2}{*}{$2,50 \pm 1,14$} & \multirow{4}{*}{$<0,001$ * } \\
\hline & $=$ pré & $4(17, .3)$ & & & \\
\hline & $>$ pré & $0 \quad(0,0)$ & \multirow{2}{*}{ pós-prótese } & \multirow{2}{*}{$0,68 \pm 0,84$} & \\
\hline & Total & $23(100,0)$ & & & \\
\hline \multirow{4}{*}{$\begin{array}{l}120^{\circ} \text { dia } \\
\text { Pós-prótese }\end{array}$} & < pré & $9(66,7)$ & \multirow{2}{*}{ pré-prótese } & \multirow{2}{*}{$2,67 \pm 0,99$} & \multirow{4}{*}{0,010 * } \\
\hline & $=$ pré & $4(33,3)$ & & & \\
\hline & $>$ pré & $0(0,0)$ & \multirow{2}{*}{ pós-prótese } & \multirow{2}{*}{$1,42 \pm 1,00$} & \\
\hline & Total & $13(100,0)$ & & & \\
\hline
\end{tabular}

Foi encontrada variação estatisticamente significante do grau de disfagia nas avaliações pós-prótese quando comparadas à avaliação pré-prótese $\left(p<0,001\right.$ no $7^{\circ}$ dia, $p<0,001$ no $30^{\circ}$ dia e $p=0,010$ no $120^{\circ}$ dia). Nas três avaliações pós-prótese, observa-se redução do grau de disfagia quando comparado ao grau pré-prótese.

Tabela 1.3.3 Avaliação do grau de dor torácica.

\begin{tabular}{|c|c|c|c|c|c|}
\hline $\begin{array}{l}\text { Avaliação de } \\
\text { dor torácica }\end{array}$ & grau pós & $n \quad(\%)$ & & média $\pm d p$ & $p$ \\
\hline \multirow{4}{*}{$\begin{array}{l}7^{\circ} \text { dia } \\
\text { Pós-prótese }\end{array}$} & $<$ pré & $1(3,6)$ & \multirow{2}{*}{ pré-prótese } & \multirow{2}{*}{$1,64 \pm 3,00$} & \multirow{4}{*}{0,011 * } \\
\hline & $=$ pré & $14(50,0)$ & & & \\
\hline & > pré & $13(46,4)$ & \multirow{2}{*}{ pós-prótese } & \multirow{2}{*}{$3,82 \pm 3,95$} & \\
\hline & Total & $28 \quad(100,0)$ & & & \\
\hline \multirow{4}{*}{$\begin{array}{l}30^{\circ} \text { dia } \\
\text { Pós-prótese }\end{array}$} & < pré & $1(4,3)$ & \multirow{2}{*}{ pré-prótese } & \multirow{2}{*}{$1,78 \pm 3,16$} & \multirow{4}{*}{0,025 * } \\
\hline & $=$ pré & $11(47,8)$ & & & \\
\hline & $>$ pré & $11(47,8)$ & \multirow{2}{*}{ pós-prótese } & \multirow{2}{*}{$3,78 \pm 3,69$} & \\
\hline & Total & $23(100,0)$ & & & \\
\hline
\end{tabular}




\begin{tabular}{|c|c|c|c|c|c|}
\hline $\begin{array}{l}\text { Avaliação de } \\
\text { dor torácica }\end{array}$ & grau pós & $\mathrm{n} \quad(\%)$ & & média $\pm d p$ & $\mathrm{p}$ \\
\hline \multirow{4}{*}{$\begin{array}{l}120^{\circ} \text { dia } \\
\text { Pós-prótese }\end{array}$} & $<$ pré & $0(0,0)$ & \multirow{2}{*}{ pré-prótese } & \multirow{2}{*}{$1,00 \pm 1,96$} & \multirow{4}{*}{0,020 * } \\
\hline & $=$ pré & $7(53,8)$ & & & \\
\hline & $>$ pré & $6(46,2)$ & \multirow{2}{*}{ pós-prótese } & \multirow{2}{*}{$3,08 \pm 3,25$} & \\
\hline & Total & $13(100,0)$ & & & \\
\hline
\end{tabular}

Aos sete dias pós-prótese, 28 pacientes foram avaliados quanto à dor torácica, onde $14(50,0 \%)$ não apresentaram alteração no escore de dor, 1 (3,6\%) apresentou redução e 14 (46,4\%) apresentaram aumento do mesmo.

A média do escore de dor pré-prótese na amostra de 28 pacientes foi de 1,64 (dp = $3,00)$ e aumentou de forma estatisticamente significante $(p=0,011)$ para $3,82(d p=3,95)$ na avaliação de sete dias pós-prótese.

Aos 30 dias pós-prótese, 23 pacientes foram avaliados quanto à presença de dor torácica, onde 11 (47,8\%) não apresentaram alteração, um (4,3\%) apresentou redução e $11(47,8 \%)$ apresentaram aumento desse sintoma.

A média do escore de dor pré-prótese na amostra de 23 pacientes foi de 1,78 (dp = $3,16)$ e aumentou de forma estatisticamente significante $(p=0,025)$ para $3,78(d p=3,69)$ na avaliação de 30 dias pós-prótese.

Aos 120 dias pós -prótese, 13 pacientes foram avaliados quanto à presença de dor torácica, onde sete $(53,8 \%)$ não apresentaram alteração e seis $(46,2 \%)$ apresentaram aumento da mesma.

A média do escore de dor pré-prótese na amostra de 13 pacientes foi de 1,00 (dp = $1,96)$ e aumentou de forma estatisticamente significante $(p=0,020)$ para $3,08(d p=3,25)$ na avaliação de 120 dias pós-prótese. 
Tabela 1.3.4 Avaliação do grau de eructação.

\begin{tabular}{|c|c|c|c|c|c|}
\hline $\begin{array}{l}\text { Avaliação de } \\
\text { eructação }\end{array}$ & grau pós & $\mathrm{n} \quad(\%)$ & & média $\pm d p$ & $p$ \\
\hline \multirow{4}{*}{$\begin{array}{l}7^{\circ} \text { dia } \\
\text { Pós-prótese }\end{array}$} & $<$ pré & $3(10,7)$ & \multirow{2}{*}{ pré-prótese } & \multirow{2}{*}{$0,82 \pm 2,11$} & \multirow{4}{*}{0,417} \\
\hline & $=$ pré & $19(67,9)$ & & & \\
\hline & $>$ pré & $6(21,4)$ & \multirow{2}{*}{ pós-prótese } & \multirow{2}{*}{$1,43 \pm 3,00$} & \\
\hline & Total & $28 \quad(100,0)$ & & & \\
\hline \multirow{4}{*}{$\begin{array}{l}30^{\circ} \text { dia } \\
\text { Pós-prótese }\end{array}$} & $<$ pré & $2(8,7)$ & \multirow{2}{*}{ pré-prótese } & \multirow{2}{*}{$0,68 \pm 1,76$} & \multirow{4}{*}{0,257} \\
\hline & $=$ pré & $16(69,5)$ & & & \\
\hline & $>$ pré & $5 \quad(21,7)$ & \multirow{2}{*}{ pós-prótese } & \multirow{2}{*}{$1,36 \pm 2,75$} & \\
\hline & Total & $23 \quad(100,0)$ & & & \\
\hline \multirow{4}{*}{$\begin{array}{l}120^{\circ} \text { dia } \\
\text { Pós-prótese }\end{array}$} & $<$ pré & $0 \quad(0,0)$ & \multirow{2}{*}{ pré-prótese } & \multirow{2}{*}{$0,38 \pm 1,39$} & \multirow{4}{*}{0,083} \\
\hline & $=$ pré & $10(76,9)$ & & & \\
\hline & $>$ pré & $3(23,1)$ & \multirow{2}{*}{ pós-prótese } & \multirow{2}{*}{$1,54 \pm 3,15$} & \\
\hline & Total & $13(100,0)$ & & & \\
\hline
\end{tabular}

Não foi encontrada variação estatisticamente significante do grau de eructação nas avaliações pós-prótese quando comparadas à avaliação pré-prótese $\left(p=0,417\right.$ no $7^{\circ}$ dia, $p=0,257$ no $30^{\circ}$ dia e $p=0,083$ no $120^{\circ}$ dia).

Tabela 1.3.5 Avaliação do grau de pirose.

\begin{tabular}{|c|c|c|c|c|c|}
\hline $\begin{array}{l}\text { Avaliação de } \\
\text { pirose }\end{array}$ & grau pós & $\begin{array}{ll}\mathrm{n} & (\%)\end{array}$ & & média $\pm d p$ & $p$ \\
\hline \multirow{4}{*}{$\begin{array}{l}7^{\circ} \text { dia } \\
\text { Pós-prótese }\end{array}$} & $<$ pré & $4(14,2)$ & \multirow{2}{*}{ pré-prótese } & \multirow{2}{*}{$0,86 \pm 2,25$} & \multirow{4}{*}{0,916} \\
\hline & $=$ pré & $22(78,5)$ & & & \\
\hline & $>$ pré & $2(7,1)$ & \multirow{2}{*}{ pós-prótese } & \multirow{2}{*}{$0,86 \pm 2,34$} & \\
\hline & Total & $28(100,0)$ & & & \\
\hline \multirow{4}{*}{$\begin{array}{l}30^{\circ} \text { dia } \\
\text { Pós-prótese }\end{array}$} & < pré & $3(13,0)$ & \multirow{2}{*}{ pré-prótese } & \multirow{2}{*}{$0,87 \pm 2,34$} & \multirow{4}{*}{0,786} \\
\hline & $=$ pré & $18 \quad(78,3)$ & & & \\
\hline & $>$ pré & $2(8,7)$ & \multirow{2}{*}{ pós-prótese } & \multirow{2}{*}{$1,09 \pm 2,59$} & \\
\hline & Total & $23(100,0)$ & & & \\
\hline
\end{tabular}




\begin{tabular}{|c|c|c|c|c|c|}
\hline $\begin{array}{l}\text { Avaliação de } \\
\text { pirose }\end{array}$ & grau pós & $\mathrm{n} \quad(\%)$ & & média $\pm d p$ & $P$ \\
\hline \multirow{4}{*}{$\begin{array}{l}120^{\circ} \text { dia } \\
\text { Pós-prótese }\end{array}$} & < pré & $1(7,7)$ & \multirow{2}{*}{ pré-prótese } & \multirow{2}{*}{$0,54 \pm 1,94$} & \multirow{4}{*}{0,655} \\
\hline & $=$ pré & $11(84,6)$ & & & \\
\hline & $>$ pré & $1(7,7)$ & \multirow{2}{*}{ pós-prótese } & \multirow{2}{*}{$0,77 \pm 1,88$} & \\
\hline & Total & $13(100,0)$ & & & \\
\hline
\end{tabular}

Pela Tabela 1.3.5, nota-se que do pré-operatório para $07^{\circ}$ dia, a proporção de pacientes com aumento da pirose não é estatisticamente significativa $(p=0,916)$. $O$ mesmo ocorre quando se avalia do pré-operatório para o $30^{\circ}$ dia $(p=0,786)$ e do préoperatório para o $120^{\circ}$ dia $(p=0,655)$.

Tabela 1.3.6 Avaliação do grau de regurgitação.

\begin{tabular}{|c|c|c|c|c|c|}
\hline $\begin{array}{l}\text { Avaliação da } \\
\text { regurgitação }\end{array}$ & grau pós & $\mathrm{n} \quad(\%)$ & & média $\pm d p$ & $P$ \\
\hline \multirow{4}{*}{$\begin{array}{l}7^{\circ} \text { dia } \\
\text { Pós-prótese }\end{array}$} & < pré & $17(60,7)$ & \multirow{2}{*}{ pré-prótese } & \multirow{2}{*}{$5,38 \pm 4,66$} & \multirow{4}{*}{0,001 * } \\
\hline & $=$ pré & $9(32,1)$ & & & \\
\hline & $>$ pré & $2(7,1)$ & \multirow{2}{*}{ pós-prótese } & \multirow{2}{*}{$0,79 \pm 2,47$} & \\
\hline & Total & $28 \quad(100,0)$ & & & \\
\hline \multirow{4}{*}{$\begin{array}{l}30^{\circ} \text { dia } \\
\text { Pós-prótese }\end{array}$} & < pré & $14(60,8)$ & \multirow{2}{*}{ pré-prótese } & \multirow{2}{*}{$5,27 \pm 4,81$} & \multirow{4}{*}{0,008 * } \\
\hline & $=$ pré & $7 \quad(30,4)$ & & & \\
\hline & $>$ pré & $2(8,7)$ & \multirow{2}{*}{ pós-prótese } & \multirow{2}{*}{$1,14 \pm 2,64$} & \\
\hline & Total & $23 \quad(100,0)$ & & & \\
\hline \multirow{4}{*}{$\begin{array}{l}120^{\circ} \text { dia } \\
\text { Pós-prótese }\end{array}$} & < pré & $7(53,8)$ & \multirow{2}{*}{ pré-prótese } & \multirow{2}{*}{$5,62 \pm 4,99$} & \multirow{4}{*}{0,039 * } \\
\hline & $=$ pré & $5(38,5)$ & & & \\
\hline & $>$ pré & $1(7,7)$ & \multirow{2}{*}{ pós-prótese } & \multirow{2}{*}{$2,31 \pm 3,30$} & \\
\hline & Total & $13(100,0)$ & & & \\
\hline
\end{tabular}

Foi encontrada variação estatisticamente significante quanto ao grau de regurgitação nas avaliações pós-prótese quando comparadas à avaliação pré-prótese $\left(p=0,001\right.$ no $7^{\circ}$ dia, 
$p=0,008$ no $30^{\circ}$ dia e $p=0,039$ no $120^{\circ}$ dia). Nas três avaliações pós- -prótese, observa-se redução do grau de regurgitação quando comparado ao grau pré-prótese.

Tabela 1.3.7 Avaliação do grau de tosse.

\begin{tabular}{|c|c|c|c|c|c|}
\hline $\begin{array}{l}\text { Avaliação da } \\
\text { tosse }\end{array}$ & grau pós & $n \quad(\%)$ & & média $\pm \mathrm{dp}$ & $\mathrm{P}$ \\
\hline \multirow{4}{*}{$\begin{array}{l}7^{\circ} \text { dia } \\
\text { pós-prótese }\end{array}$} & < pré & $9(32,1)$ & \multirow{2}{*}{ pré-prótese } & \multirow{2}{*}{$2,21 \pm 3,04$} & \multirow{4}{*}{0,410} \\
\hline & $=$ pré & $14(50,0)$ & & & \\
\hline & $>$ pré & $5(17,8)$ & \multirow{2}{*}{ pós-prótese } & \multirow{2}{*}{$1,66 \pm 2,98$} & \\
\hline & Total & $28 \quad(100,0)$ & & & \\
\hline \multirow{4}{*}{$\begin{array}{l}30^{\circ} \text { dia } \\
\text { pós-prótese }\end{array}$} & $<$ pré & $5 \quad(21,7)$ & \multirow{2}{*}{ pré-prótese } & \multirow{2}{*}{$1,95 \pm 2,85$} & \multirow{4}{*}{0,602} \\
\hline & $=$ pré & $13(56,5)$ & & & \\
\hline & $>$ pré & $5(21,7)$ & \multirow{2}{*}{ pós-prótese } & \multirow{2}{*}{$2,27 \pm 3,27$} & \\
\hline & Total & $23(100,0)$ & & & \\
\hline \multirow{4}{*}{$\begin{array}{l}120^{\circ} \text { dia } \\
\text { pós-prótese }\end{array}$} & $<$ pré & $3(23,1)$ & \multirow{2}{*}{ pré-prótese } & \multirow{2}{*}{$2,00 \pm 2,48$} & \multirow{4}{*}{0,888} \\
\hline & $=$ pré & $8(61,5)$ & & & \\
\hline & $>$ pré & $2(15,4)$ & \multirow{2}{*}{ pós-prótese } & \multirow{2}{*}{$1,92 \pm 2,53$} & \\
\hline & Total & $13(100,0)$ & & & \\
\hline
\end{tabular}

Não foi encontrada variação estatisticamente significante do grau de tosse nas avaliações pós-prótese quando comparadas à avaliação pré-prótese $\left(p=0,410\right.$ no $7^{\circ}$ dia, $p=0,602$ no $30^{\circ}$ dia e $p=0,888$ no $\left.120^{\circ} \mathrm{dia}\right)$.

Tabela 1.3.8 Resultados dos testes do sinal para avaliar o índice de Karnofskycondição global (lkc)

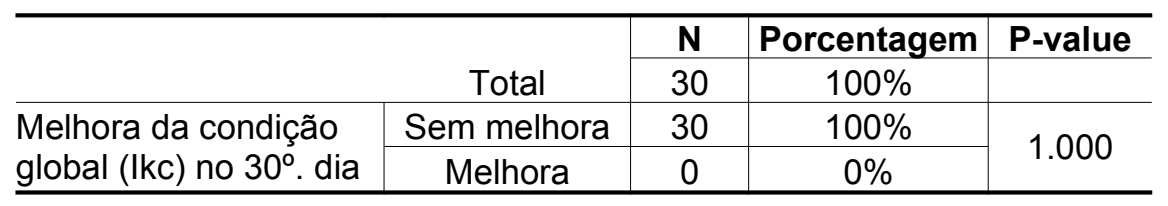

Pela Tabela 1.3.8, é possível observar que do dia pré-procedimento até o $30^{\circ}$ dia não houve melhora na condição global do paciente. 
Tabela 1.3.9 Resultados dos testes do sinal para avaliar o índice de Karnofskyaptidão física (Ikp)

\begin{tabular}{lcccc}
\hline & & $\mathbf{N}$ & Porcentagem & P-value \\
& Total & 30 & $100 \%$ & \\
Melhora da aptidão & Sem melhora & 22 & $73,3 \%$ & \multirow{2}{*}{0.53} \\
física (Ikp) no 30 $30^{\circ}$. dia & Melhora & 8 & $26,6 \%$ & \\
\hline
\end{tabular}

Pela Tabela 1.3.9, observa-se que do pré-procedimento até o $30^{\circ}$ dia não há melhora na aptidão física do paciente.

\subsection{Exames complementares}

Exame radiológico contrastado do esôfago e estômago pós-procedimento: foram realizados 25 exames radiológicos com bário no sétimo dia pós-procedimento. Nenhum deles apresentou refluxo do contraste pela válvula, mesmo em posição de Trendelemburg, mostrando um bom fluxo anterógrado.

Endoscopia digestiva alta pós-procedimento: foi realizada em 27 dos 30 pacientes, com diagnóstico de seis rupturas de válvulas, quatro hiperplasias teciduais nas extremidades da prótese e quatro migrações distais.

\subsection{Complicações}

\begin{tabular}{lcc}
\hline Complicações & $\mathrm{n}$ & $\%$ \\
\hline Perfuração & 3 & 10,0 \\
Hemorragia & 7 & 23,3 \\
Migração da prótese & 4 & 13,3 \\
Ruptura da válvula & 6 & 20,0 \\
Hiperplasia tecidual benigna & 4 & 13,3 \\
\hline
\end{tabular}

Dos 30 pacientes da amostra, $13(43,3 \%)$ não apresentaram nenhuma complicação e 17 $(56,7 \%)$ apresentaram, pelo menos uma daquelas relacionadas na tabela acima. 


\subsubsection{Associação entre TNM e Complicações}

\begin{tabular}{|c|c|c|c|c|c|}
\hline \multirow{2}{*}{$\begin{array}{l}\text { Complicações } \\
\text { Perfuração - n (\%) }\end{array}$} & \multicolumn{2}{|c|}{$\begin{array}{l}\text { TNM III } \\
(n=5)\end{array}$} & \multicolumn{2}{|c|}{$\begin{array}{l}\text { TNM IV } \\
(n=25)\end{array}$} & \multirow[t]{2}{*}{$\begin{array}{c}\text { Teste exato } \\
\text { de Fisher }\end{array}$} \\
\hline & & & & & \\
\hline Ausente & 5 & $100,0 \%$ & 22 & $88,0 \%$ & \multirow{2}{*}{$p=1,000$} \\
\hline Presente & 0 & $0,0 \%$ & 3 & $12,0 \%$ & \\
\hline \multicolumn{6}{|c|}{ Hemorragia - n (\%) } \\
\hline Ausente & 4 & $80,0 \%$ & 19 & $76,0 \%$ & \multirow{2}{*}{$p=1,000$} \\
\hline Presente & 1 & $20,0 \%$ & 6 & $24,0 \%$ & \\
\hline \multicolumn{6}{|c|}{ Migração da prótese - n (\%) } \\
\hline Ausente & 3 & $60,0 \%$ & 23 & $92,0 \%$ & \multirow{2}{*}{$p=0,119$} \\
\hline Presente & 2 & $40,0 \%$ & 2 & $8,0 \%$ & \\
\hline \multicolumn{6}{|c|}{ Ruptura da válvula - n (\%) } \\
\hline Ausente & 2 & $40,0 \%$ & 22 & $88,0 \%$ & \multirow{2}{*}{$p=0,041$ * } \\
\hline Presente & 3 & $60,0 \%$ & 3 & $12,0 \%$ & \\
\hline \multicolumn{6}{|c|}{ Hiperplasia tecidual benigna - n (\%) } \\
\hline Ausente & 4 & $80,0 \%$ & 22 & $88,0 \%$ & \multirow{2}{*}{$p=0,538$} \\
\hline Presente & 1 & $20,0 \%$ & 3 & $12,0 \%$ & \\
\hline
\end{tabular}

Demonstrou-se uma associação estatisticamente significante entre os graus da classificação TNM e a presença de ruptura da válvula $(p=0,041)$, onde no grupo de pacientes com TNM estádio III, 60\% deles apresentaram ruptura da válvula e no grupo TNM estádio IV essa proporção foi significativamente mais baixa, presente em apenas $12 \%$ dos pacientes.

Não houve associação estatisticamente significante entre o tipo de estádio TNM e as demais complicações ( $p>0,05$ em todas as análises). 


\subsubsection{Sobrevida}

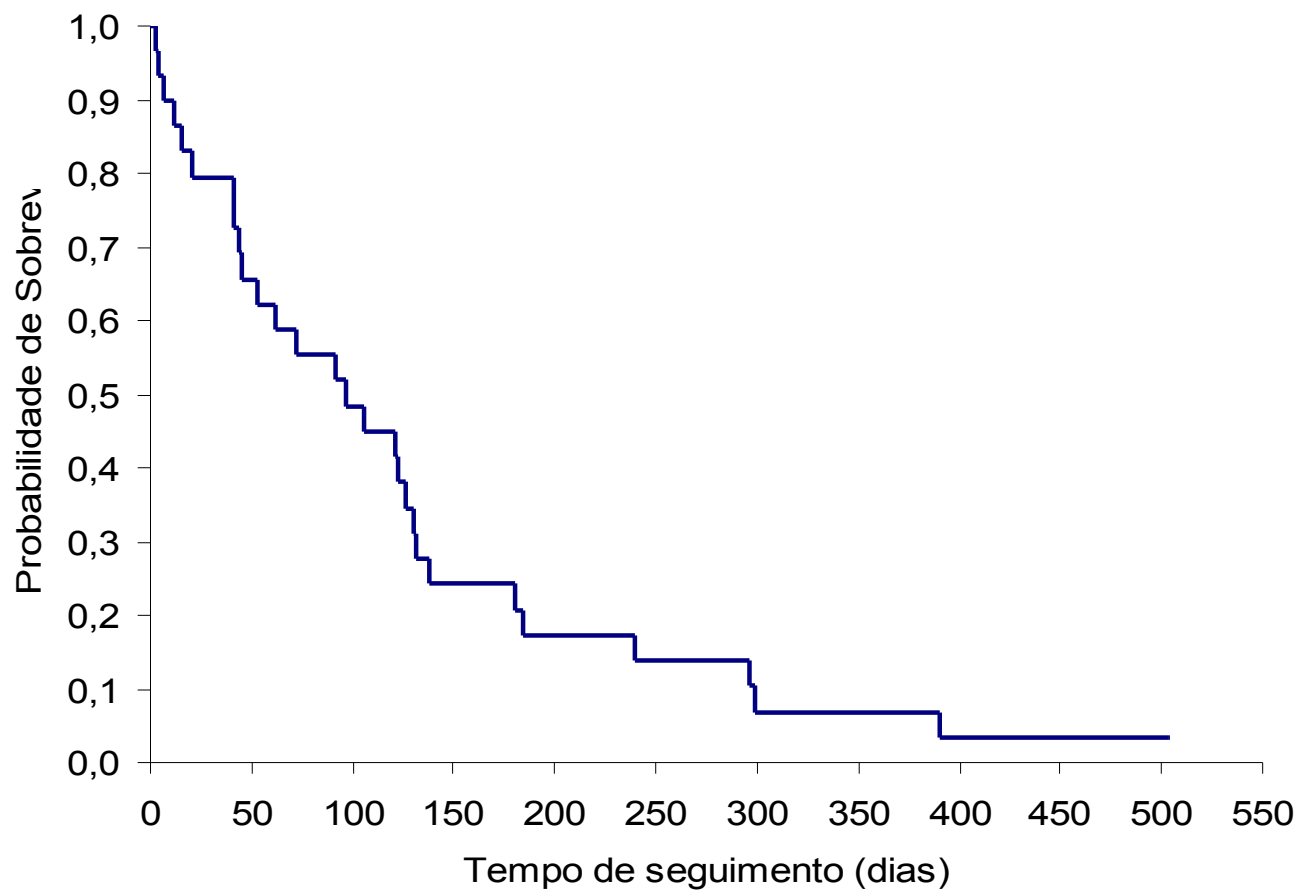

\section{Gráfico 1.5.2 Probabilidade de sobrevida $\mathrm{x}$ tempo de seguimento}

Dos 30 pacientes avaliados, 28 (93,3\%) obituaram durante o seguimento, dois permaneceram vivos até o final do estudo.

A média do tempo de sobrevida foi de 124 dias (ep = 22 dias) com intervalo com $95 \%$ de confiança: [80; 167].

\subsubsection{Associação entre complicações x sobrevida}

A igualdade das distribuições de sobrevida dos pacientes foi testada em relação a cada uma das complicações como perfuração, hemorragia e hiperplasia tecidual benigna, cujos resultados estão apresentados na tabela 1.5.2.1 e gráficos 1.5.2.1a, b e c. 
Tabela 1.5.2.1 Complicações x sobrevida

\begin{tabular}{|c|c|c|c|c|c|c|c|}
\hline Complicações & Casos & & ventos & Média & ep & $\mathrm{IC}_{95 \%}$ & $\begin{array}{l}\text { Teste log } \\
\text { rank }\end{array}$ \\
\hline \multicolumn{8}{|l|}{ Perfuração } \\
\hline Ausente & 27 & 25 & $92,6 \%$ & 136 & 24 & {$[90 ; 182]$} & \multirow{2}{*}{$p<0,001$ * } \\
\hline Presente & 3 & 3 & $100,0 \%$ & 17 & 12 & {$[0 ; 41]$} & \\
\hline \multicolumn{8}{|l|}{ Hemorragia } \\
\hline Ausente & 23 & 21 & $91,3 \%$ & 130 & 28 & {$[75 ; 185]$} & \multirow{2}{*}{$p=0,564$} \\
\hline Presente & 7 & 7 & $100,0 \%$ & 103 & 25 & {$[53 ; 152]$} & \\
\hline \multicolumn{8}{|c|}{ Hiperplasia tecidual benigna } \\
\hline Ausente & 26 & 25 & $96,1 \%$ & 108 & 21 & {$[67 ; 149]$} & \multirow{2}{*}{$p=0,122$} \\
\hline Presente & 4 & 3 & $75,0 \%$ & 223 & 81 & {$[64 ; 381]$} & \\
\hline
\end{tabular}

A presença de perfuração foi associada à menor média de sobrevida, com correlação estatisticamente significante $(p<0,001)$.

Não houve relação estatisticamente significante entre a incidência de hemorragia $(p=$ $0,564)$ e hiperplasia tecidual benigna $(p=0,122)$ com a sobrevida dos pacientes.

Os gráficos a seguir representam as curvas de sobrevida dos pacientes segundo a presença de complicações. 


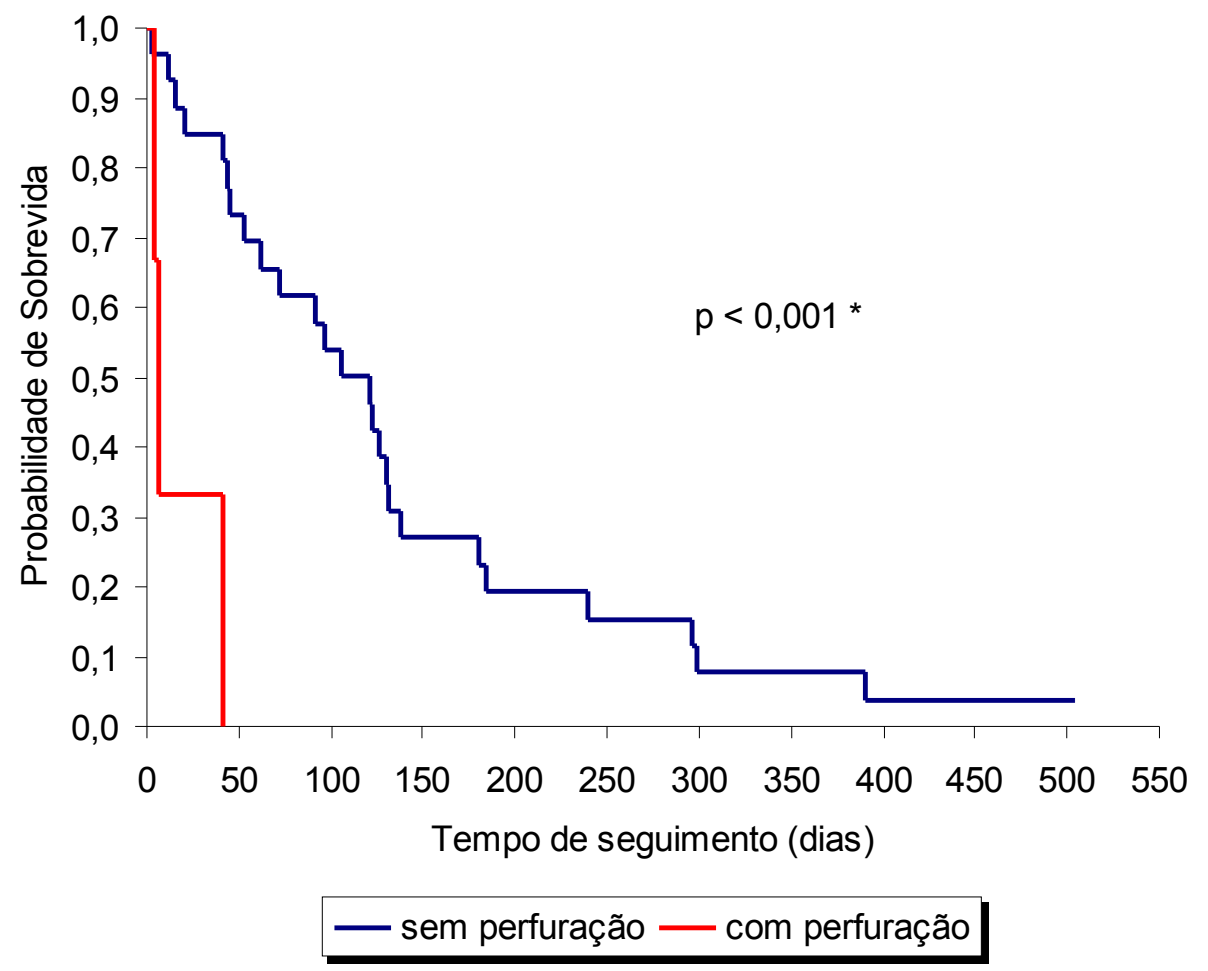

Gráfico 1 5.2.1a Perfuração x probabilidade de sobrevida

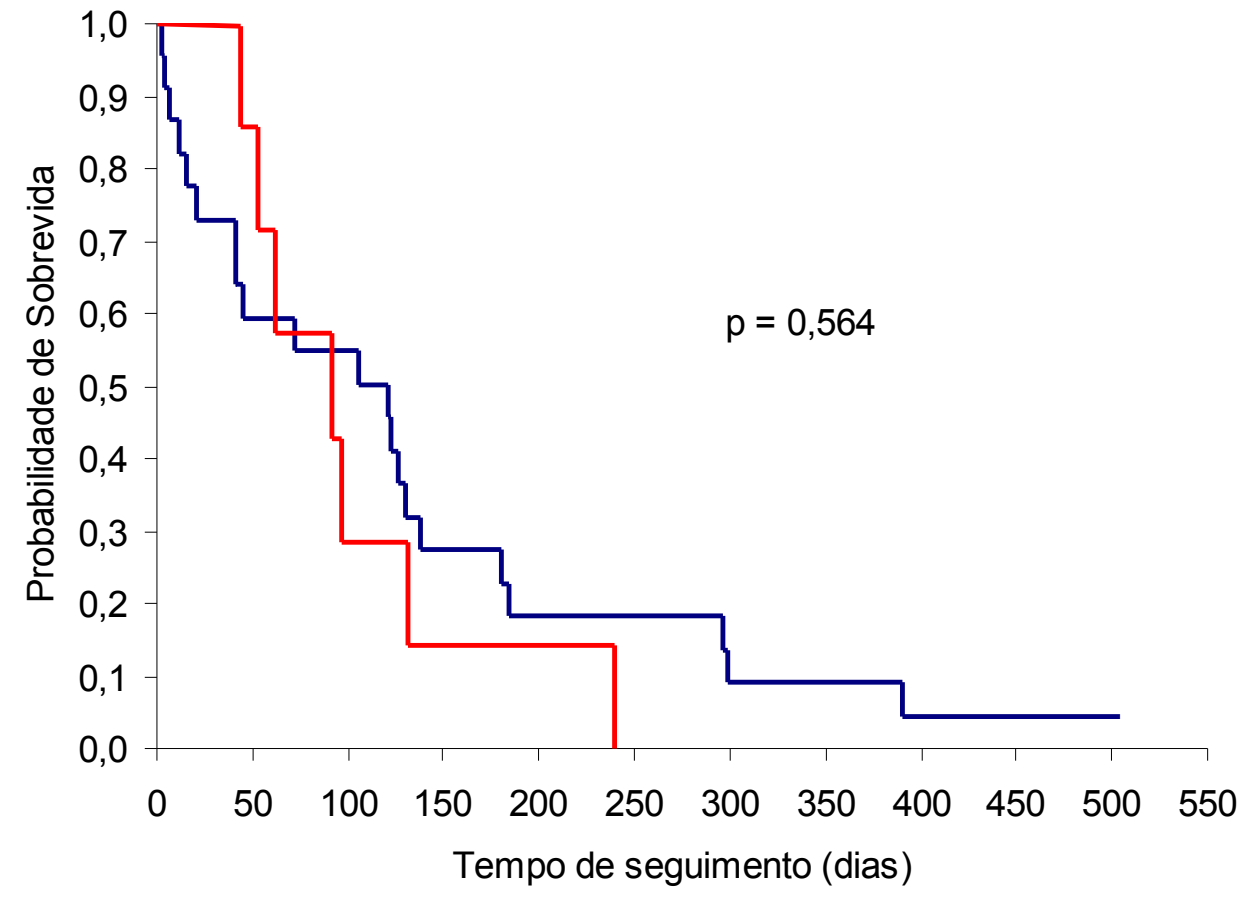

— sem hemorragia —_ com hemorragia

Gráfico 1.5.2.1b Hemorragia x probabilidade de sobrevida 


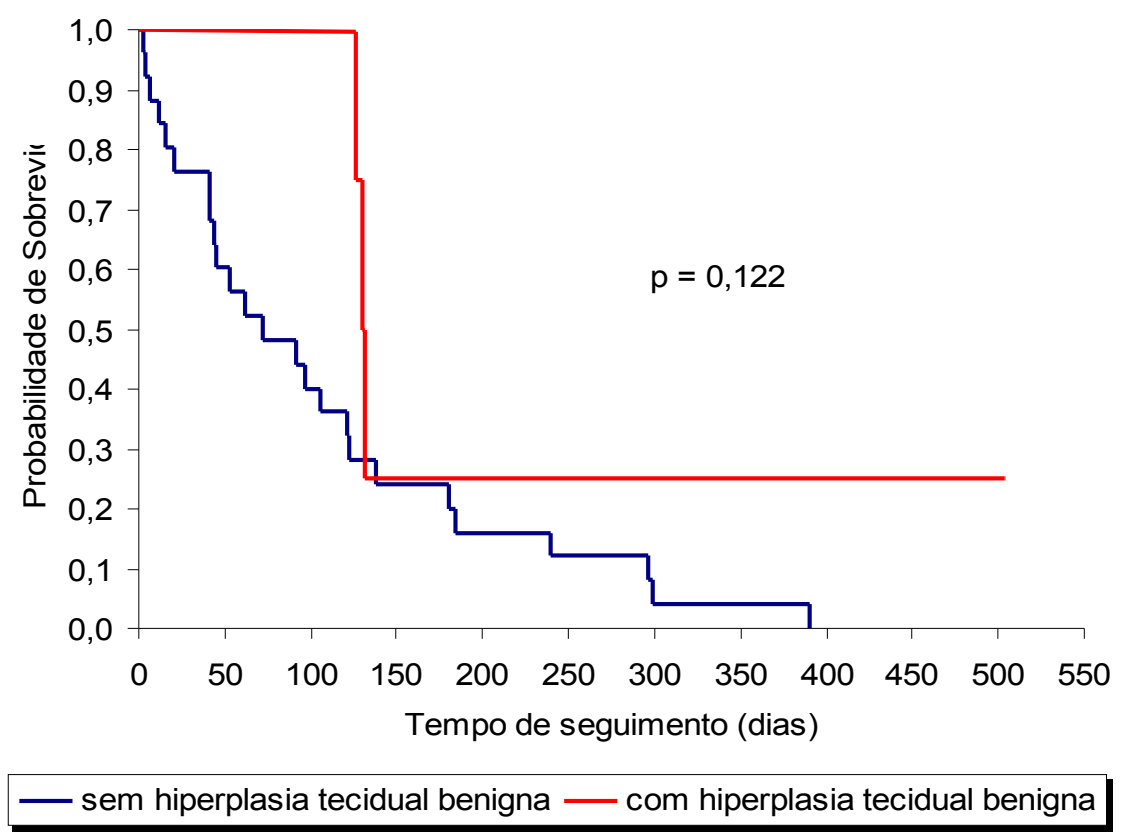

Gráfico 1.5.2.1c Hiperplasia tecidual benigna x probabilidade de sobrevida

1.5.3 Associação entre Quimioterapia (QT) e/ou radioterapia (RT) e Complicações Tabela 1.5.3 Complicações x QT/RT

\begin{tabular}{|c|c|c|c|c|c|}
\hline \multirow{2}{*}{$\begin{array}{l}\text { Complicações } \\
\text { Perfuração - n (\% }\end{array}$} & \multicolumn{2}{|c|}{$\begin{array}{c}\text { Sem QT e } \\
\text { sem RT }(n= \\
26)\end{array}$} & \multicolumn{2}{|c|}{$\begin{array}{c}\text { Com QT elou } \\
\text { com RT }(n= \\
4)\end{array}$} & \multirow[t]{2}{*}{$\begin{array}{c}\text { Teste exato } \\
\text { de Fisher }\end{array}$} \\
\hline & & & & & \\
\hline Ausente & 24 & $92,3 \%$ & 3 & $75,0 \%$ & \multirow{2}{*}{$p=0,360$} \\
\hline Presente & 2 & $7,7 \%$ & 1 & $25,0 \%$ & \\
\hline \multicolumn{6}{|c|}{ Hemorragia - n (\%) } \\
\hline Ausente & 21 & $80,8 \%$ & 2 & $50,0 \%$ & \multirow{2}{*}{$p=0,225$} \\
\hline Presente & 5 & $19,2 \%$ & 2 & $50,0 \%$ & \\
\hline \multicolumn{6}{|c|}{ Migração da prótese - n (\%) } \\
\hline Ausente & 22 & $84,6 \%$ & 4 & $100,0 \%$ & \multirow{2}{*}{$p=1,000$} \\
\hline Presente & 4 & $15,4 \%$ & 0 & $0,0 \%$ & \\
\hline \multicolumn{6}{|c|}{ Ruptura da válvula - n (\%) } \\
\hline Ausente & 20 & $76,9 \%$ & 4 & $100,0 \%$ & \multirow{2}{*}{$p=0,557$} \\
\hline Presente & 6 & $23,1 \%$ & 0 & $0,0 \%$ & \\
\hline Complicações & \multicolumn{2}{|c|}{$\begin{array}{c}\text { Sem QT e } \\
\text { sem RT }(n= \\
26)\end{array}$} & \multicolumn{2}{|c|}{$\begin{array}{c}\text { Com QT e/ou } \\
\text { com RT }(n= \\
4)\end{array}$} & $\begin{array}{c}\text { Teste exato } \\
\text { de Fisher }\end{array}$ \\
\hline
\end{tabular}


Hiperplasia tecidual benigna $-\mathrm{n}$
(\%)
Ausente
$2284,6 \%$
$4100,0 \%$
Presente
$4 \quad 15,4 \% \quad 0 \quad 0,0 \%$
$p=1,000$

Não houve associação estatisticamente significante entre o uso de QT e/ou RT e a ocorrência de complicações ( $p>0,05)$ em todas as análises.

\subsubsection{Associação entre Tu circunferencial e migração da prótese}

Tabela 1.5.4 Migração da prótese x característica do tumor

\begin{tabular}{lccccc}
\hline Complicação & $\begin{array}{c}\text { Tu não } \\
\text { circunferencial } \\
(\mathbf{n}=\mathbf{3})\end{array}$ & $\begin{array}{c}\text { Tu } \\
\text { circunferencial } \\
(\mathbf{n}=\mathbf{2 7})\end{array}$ & $\begin{array}{c}\text { Teste exato } \\
\text { de Fisher }\end{array}$ \\
\hline Migração da prótese $-\mathrm{n}(\%)$ & 2 & $66,7 \%$ & 24 & $88,9 \%$ & $\mathrm{p}=0,360$ \\
Ausente & 1 & $33,3 \%$ & 3 & $11,1 \%$ & \\
Presente & & & & &
\end{tabular}

Não se observou associação estatisticamente significante entre o envolvimento neoplásico circunferencial ou não e a ocorrência de migração da prótese $(p=0,360)$.

1.5.5 Associação entre ruptura da válvula e sintomas de refluxo gastroesofágico

Tabela 1.5.5 Ruptura da válvula x sintomas de RGE.

\begin{tabular}{cc}
\hline Associação & p-value \\
\hline Pirose X Ruptura da válvula & 1.000 \\
Regurgitação X Ruptura da válvula & $\mathbf{0 . 0 0 5}$ \\
Tosse X Ruptura da válvula & 0.592 \\
Eructação X Ruptura da válvula & 0.070 \\
\hline
\end{tabular}


Pela Tabela 1.5.5, nota-se que a ruptura da válvula correlacionou-se com a presença de regurgitação $(p=0,005)$ enquanto os demais sintomas de refluxo gastroesofágico não tiveram essa associação. 
Discussão 


\section{Discussão}

O tratamento atual do câncer de esôfago apresenta abordagem mais individualizada, sendo primordial o estadiamento da doença. A mortalidade operatória de $72 \%$, descrita por Oschner e DeBakey em 1940, caiu para 29\% na década de $70,13 \%$ nos anos 80 e, a partir dos anos 90, a mortalidade em países desenvolvidos corresponde a $11 \%$. Em centros de referência, pode-se obter uma taxa de mortalidade operatória inferior a $5 \%$ (LAW S, 2004).

O carcinoma epidermóide (CEC) e o adenocarcinoma (ADC) são os dois tipos histológicos mais freqüentes que acometem o terço distal do esôfago. O CEC incide em população com média de idade menor que o ADC, apresenta maior relação entre homem:mulher (8:1 vs 4:1), associa-se com hábitos de tabagismo e consumo de álcool. Todavia, o tipo histológico não é fator determinante do tempo de sobrevida, sendo o estágio da doença, no momento do diagnóstico, mais relevante que o tipo celular (ALEXANDROU A, 2002).

O aumento na incidência, nos países ocidentais, do adenocarcinoma de cárdia, observado a partir de 1970, tem sido relacionado a diversos fatores: o refluxo gastroesofágico, o aumento no índice de massa corpórea, a presença do Esôfago de Barrett, a maior precisão nos métodos de diagnóstico e a adoção de uma classificação para diferenciar os tumores de origem esofágica e gástrica ( CAMERON AJ, 2002; LAGERGREEN J, 1999; SPECHLER SJ, 2003; WALTER C, 2004; WEI JT, 2003). A incidência de adenocarcinoma em pacientes com Esôfago de Barrett é de aproximadamente $0,5 \%$ ao ano, sendo que, além do refluxo gastroesofágico, a raça 
branca, o sexo masculino, a idade avançada e a obesidade são fatores predisponentes (SPECHLER SJ, 2003).

A endoscopia digestiva alta é o método de diagnóstico fundamental, pelo qual a neoplasia é identificada sob visão direta e colhido material para histologia. O uso de corantes, entre eles o lugol e o azul de metileno, facilita a visibilização de lesões que não são aparentes à endoscopia sem cromoscopia. Novas tecnologias como a endoscopia de alta resolução, autofluorescência, espectroscopia, tomografia de coerência óptica, têm o objetivo de aumentar a detecção de lesões durante o exame endoscópico (MORETO M, 2005; WANG KK, 2004), porém sua utilização encontra-se em fase de experimentação clínica em centros especializados.

A ecoendoscopia é o exame complementar que apresenta maior acurácia para determinar a profundidade de invasão tumoral, ou seja, o estadiamento T da classificação TNM, com precisão de até $85 \%$, sendo que para lesões T1 e T2, quando se utiliza miniprobes de alta freqüência (15-30 MHz), a acurácia é de 83-92\%. Também é o melhor exame para definir o comprometimento ganglionar regional ( $\mathrm{N}$ da classificação $\mathrm{TNM}$ ), cuja acurácia varia de $75-80 \%$, sendo maior quando associada à punção com agulha fina e superior à tomografia computadorizada, que apresenta resultados em torno de $50 \%$, tanto para o estadiamento T, como N. A tomografia computadorizada helicoidal, por sua vez, apresenta melhores resultados na detecção de metástases à distância (JACOBSON BC, 2003; PENMAN ID, 2002).

A abordagem cirúrgica para o câncer que acomete a região da JEG também é individualizada, e baseia-se na topografia da lesão, que pode ser de três tipos: IAdenocarcinoma do esôfago distal, que se origina de uma área de metaplasia intestinal especializada (esôfago de Barrett), e que pode envolver a JEG pela porção superior; II- 
Adenocarcinoma do cárdia propriamente dito, originando-se diretamente da JEG; IIICarcinoma gástrico subcárdico, que infiltra a JEG pela porção inferior, segundo a classificação de Siewert (SALLUM RA, 1996; SIEWERT JR, 1987). As vias de acesso cirúrgico para ressecção esofágica e o estômago proximal em bloco para as lesões tipo I variam entre a toracotomia, a operação de Ivor Lewis (1946) ou a laparotomia com frenotomia mediana (PINOTTI, 1978). Nas lesões tipo III, recomenda-se a gastrectomia total associada à esofagectomia distal ou subtotal, por laparotomia e frenotomia mediana. Nas lesões tipo II, as ressecções variam conforme a extensão da lesão, e nesses casos pode-se associar a esofagectomia distal ou subtotal com a gastrectomia proximal ou total, freqüentemente por laparotomia e frenotomia mediana (ADEFNA PEREZ RI, 2004; PINOTTI HW, 1978; SALLUM RA, 1996; SIEWERT JR, 1987). As opções de reconstrução do trânsito são com o estômago remanescente nos casos de gastrectomia proximal, utilizando-se o intestino delgado ou o cólon, com anastomose por cervicotomia ou torácica alta.

O fator prognóstico primordial na obtenção da cura é a ressecção completa da neoplasia e de todos os linfonodos comprometidos. Os tumores de esôfago torácico apresentam melhor prognóstico com ressecção local da lesão e dissecção linfonodal em três campos (abdominal, torácico e cervical) (LERUT T, 2003; ALTORKI N, 2002; UDAGAWA H, 2001).

Em pacientes com tumores de esôfago distal e cárdia, a abordagem transtorácica, que proporciona dissecção locoregional mais radical, é comparada em vários estudos com a ressecção transhiatal. Em estudo retrospectivo (JOHANSSON J, 2004), analisando dois grupos de pacientes com adenocarcinoma de esôfago distal T3N1 pela classificação TNM, submetidos à ressecção em bloco transtorácica e transhiatal, demonstrou que a 
sobrevida foi influenciada por dois fatores independentes: o tipo de ressecção e o número de linfonodos envolvidos pela neoplasia. O grupo com ressecção transtorácica apresentou maior tempo de sobrevida, no entanto esse fato só foi observado em pacientes com até nove linfonodos comprometidos. Com relação à qualidade de vida após a cirurgia transtorácica e transhiatal, não há diferença entre ambas (de BOER AGEM, 2004), e a recuperação gradual do nível de atividade física ocorre no primeiro ano pós-cirurgia. A localização do adenocarcinoma, seja no esôfago distal ou no cárdia, não é fator prognóstico independente da sobrevida após a ressecção cirúrgica, e por isso é sugerido que seja considerado como entidade única (WIJNHOVEN BPL, 1999). Além do número e do local dos linfonodos comprometidos, o envolvimento extracapsular do linfonodo é indicador de pior prognóstico (LERUT T, 2003) .

Nos pacientes sem chances de cura, ou seja, na impossibilidade de ressecção completa da neoplasia maligna, a disfagia progressiva é o sintoma incapacitante mais comum, acelerando o processo de desnutrição e caquexia. Entre as terapias paliativas para a disfagia, destacam-se as cirurgias de derivação, o uso de sondas de nutrição, a radioterapia (RT), a quimioterapia (QT) e os métodos endoscópicos terapêuticos tais como: as dilatações, a permeação da lesão pela injeção de substâncias químicas, métodos ablativos e a colocação de próteses esofágicas plásticas ou metálicas (ALLUM WH, 2002; ELLIS HF, 1983; KUBBA AK, 2000; MOURA EGH, 2000).

As cirurgias de derivação, mormente as que utilizam o tubo gástrico da grande curvatura, que é de fácil confecção, apresentam um baixo índice de mortalidade e resultados satisfatórios na melhora da disfagia. Em estudo comparativo com uso de prótese metálica auto-expansível tipo InStent $₫$, os resultados foram similares, porém com maior período de hospitalização e custo final superior no grupo cirúrgico, segundo Moura 
(2000). Aoki et al. (2001) demonstraram melhores resultados na paliação da disfagia, morbidade e mortalidade utilizando o modelo Ultraflex®, quando comparado com cirurgia derivativa.

O uso de sondas de nutrição (enteral, gastrostomia, jejunostomia) é empregado como terapia auxiliar no preparo nutricional para o procedimento cirúrgico ou como método paliativo definitivo e, nesse caso, a ingestão oral não é restabelecida; a sua utilização interfere na vida social do paciente e exige cuidados no seu manuseio.

O alívio da disfagia com a RT e QT é obtido em ambos os tipos histológicos, apesar de o adenocarcinoma apresentar menor sensibilidade do que o carcinoma epidermóide. Porém, os resultados só são evidentes após algumas semanas, exigindo maior tempo de hospitalização, com duração de apenas alguns meses. Os estudos sugerem maior tempo de sobrevida dos pacientes com adenocarcinoma quando se utilizou QT (principalmente a Cisplatina e o 5-Fluorouracil) e RT neoadjuvante prévia à ressecção cirúrgica. A QT também é utilizada para tratamento paliativo na doença sistêmica metastática com remissão parcial, em 20 a 50\% dos pacientes. Nos pacientes com disfagia ou sangramento, a radioterapia externa e a braquiterapia são opções de tratamento (ALLUM WH, 2002; JACOBSON BC, 2003; MOURA EGH, 2000; RICHEL DJ, 2004).

O tratamento paliativo pela endoscopia também oferece várias alternativas para o alívio da disfagia. A mais simples é a dilatação da lesão com o uso de balões ou sondas de dilatação, porém a paliação é de curta duração, e são necessárias dilatações semanais. Esse tipo de tratamento encontra indicação nos casos de pacientes com expectativa de sobrevida muito curta, menor que quatro semanas, ou nas situações de preparação para um tratamento definitivo. 
A permeação da lesão com o uso de injeção de substâncias químicas como o álcool absoluto em alíquotas de 0,5 a $1 \mathrm{ml}$ no interior da lesão, cujo resultado final é a necrose da massa tumoral, requer sessões repetidas a cada três a sete dias. É indicada para lesões exofíticas e de consistência amolecida, não circunferenciais, próximas ao cricofaríngeo, ou para tratamento de crescimento tumoral nas extremidades da prótese (ALLUM WH, 2002; JACOBSON BC, 2003; KUBBA AK, 2000; LEE SH, 2001; LEIPER K, 2002). A injeção de quimioterápicos, como o gel de cisplatina e epinefrina, cujo objetivo é manter a ação local do quimioterápico, evita os efeitos sistêmicos da QT. Alguns estudos apresentam resultados satisfatórios na paliação da disfagia, contudo, não é isenta de complicações e, até o momento, não existem estudos controlados e com maior número de pacientes (HARBORD M, 2002; MONGA SPS, 2000).

Entre os métodos ablativos destacam-se a eletrocoagulação, a terapia fotodinâmica, a coagulação com plasma de argônio e o laser. A eletrocoagulação mono e a bipolar não são utilizadas com freqüência em virtude do difícil controle na profundidade tecidual, da necessidade de dilatação prévia e de sessões repetidas. Ela está associada a altos índices de perfuração e requer a presença de lesões circunferenciais, uma vez que a sua energia se dispersa de forma radial.

A terapia fotodinâmica consiste na administração de agente fotossensibilizante através da via sistêmica, que irá se concentrar preferencialmente no tecido tumoral. A reação fotoquímica ocorre após a exposição da lesão à radiação luminosa guiada por endoscopia, com penetração de aproximadamente $5 \mathrm{~mm}$ de profundidade, induzindo à paliação comparável ao laser, porém com o inconveniente de o agente fotossensibilizante acumular-se no organismo por várias semanas, podendo causar queimaduras cutâneas graves, caso haja exposição à luz solar. 
A coagulação com plasma de argônio é um tipo de eletrocoagulação monopolar que utiliza o gás argônio ionizado. A sua profundidade de penetração não ultrapassa 2 a 3 $\mathrm{mm}$, por isso seus resultados não são duradouros. Pode ser utilizada em pequenas lesões e no crescimento tumoral nas extremidades das próteses.

A utilização do laser, cujo tipo mais empregado é o Nd-YAG laser (Neodymium: yttrium-aluminum-garnet), é a forma mais eficaz de ablação térmica, e resulta na coagulação e vaporização do tecido neoplásico, cujo efeito é o alívio mais prolongado da disfagia. A aplicação é feita por duas técnicas: retrógrada, na qual é realizada dilatação prévia do tumor para passagem do endoscópio e aplicado o laser da extremidade distal para proximal da lesão, e a anterógrada, com aplicação do laser da extremidade proximal para a distal, sem dilatação prévia, o que limita a extensão do segmento tratado, devido ao acúmulo de tecidos necróticos na luz esofágica e edema tecidual. Apesar de baixos índices de complicação, são descritos pneumomediastino, perfuração, hemorragia e broncoaspiração. Está mais indicado nas lesões exofíticas, não circunferenciais, do esôfago e cárdia (KUBBA AK, 2000). Pode ser associado à braquiterapia de forma segura, aumentando a duração da paliação da disfagia, no entanto, sem prolongar a sobrevida (SPENCER GM, 2002).

A tunelização por próteses é opção vantajosa nas lesões estenosantes, circunferenciais, distantes mais de $2 \mathrm{~cm}$ do cricofaríngeo e de consistência firme, por proporcionar alívio imediato da disfagia em um único procedimento.

As atuais próteses metálicas auto-expansíveis apresentam sistemas introdutores de calibre reduzido, atingem diâmetros maiores com melhor paliação da disfagia e têm menor índice de complicações agudas e mortalidade, comparativamente às próteses plásticas utilizadas no passado. São indicadas para pacientes com estenoses neoplásicas 
que apresentam fístulas esofagotraqueobrônquica, com eficácia em torno de $70 \%$ (ALLUM WH, 2002; BARON TH, 1999; DUA KS, 2001; ENZINGER PC, 2003; KUBBA AK, 2000; LAW S, 2004; MONGA SPS, 2000; MOSCA F, 2003; SIEWERT JR, 2000; TYTGAT GNJ, 2004). Ferrari et al. (1997), publicaram estudo quanto à utilização de prótese metálica auto-expansível de fabricação brasileira para tratamento de CEC de esôfago complicado com fístula esofagotraqueal, vislumbrando a redução no custo da prótese de 25 a $62,5 \%$.

Com o crescente aumento na incidência do carcinoma envolvendo o esôfago distal e/ou cárdia gástrico, essas próteses são cada vez mais introduzidas através da JEG. Freqüentemente, sintomas de refluxo gastroesofágico são descritos como complicações das mesmas, e medicações supressoras de ácido são prescritas para esses pacientes, mas além de aumentarem os custos, não previnem a regurgitação e a aspiração (DUA KS, 2001; MAY A, 1995; WESTON AP, 1999).

Atualmente, as modalidades de maior relevância no tratamento paliativo das neoplasias que envolvem a junção esofagogástrica são a utilização de próteses metálicas auto-expansíveis e a permeação da lesão com o uso do laser. A destruição do mecanismo de contenção que impede o refluxo do conteúdo gástrico para o esôfago é uma preocupação constante no tratamento dessas lesões (DUA KS, 2001).

Quando uma prótese é liberada através da JEG, o estômago e o esôfago se tornam uma cavidade única. Além do refluxo de "estresse", isso é, refluxo resultante do aumento da pressão intra-abdominal, esses pacientes também podem apresentar refluxo "passivo", que ocorre quando a gravidade é eliminada, como por exemplo ao deitarem-se. Weston e Sharma (1999) relataram doença do refluxo gastroesofágico, incluindo aspiração e óbito, em $27 \%$ dos pacientes onde PMAE foram colocadas através da JEG. 
Em revisão de casos com obstrução esofágica benigna, Sandha e Marcon (1999) descreveram $21 \%$ de pacientes com sintomas de refluxo gastroesofágico relacionado a PMAE. Contudo, se as obstruções proximais e médias do esôfago fossem excluídas dessa análise, ou seja, se fossem consideradas apenas as próteses transcárdicas, a porcentagem seria acima de $40 \%$.

Algumas tentativas foram realizadas no passado para desenvolver próteses esofágicas com mecanismo anti-refluxo. Nunes et al. (1999) e Valbuena (1984) fixaram "mangas" de látex em próteses esofágicas rígidas e Mizumoto et al. (1997) usou uma modificação gorotex cilíndrica para prevenir refluxo.

No presente estudo, foi utilizado prótese metálica auto-expansível esofágica ZStent, modificada pela extensão do revestimento de poliuretano além da última gaiola metálica, com o objetivo de produzir uma válvula tipo "manga" (DUA KS, 


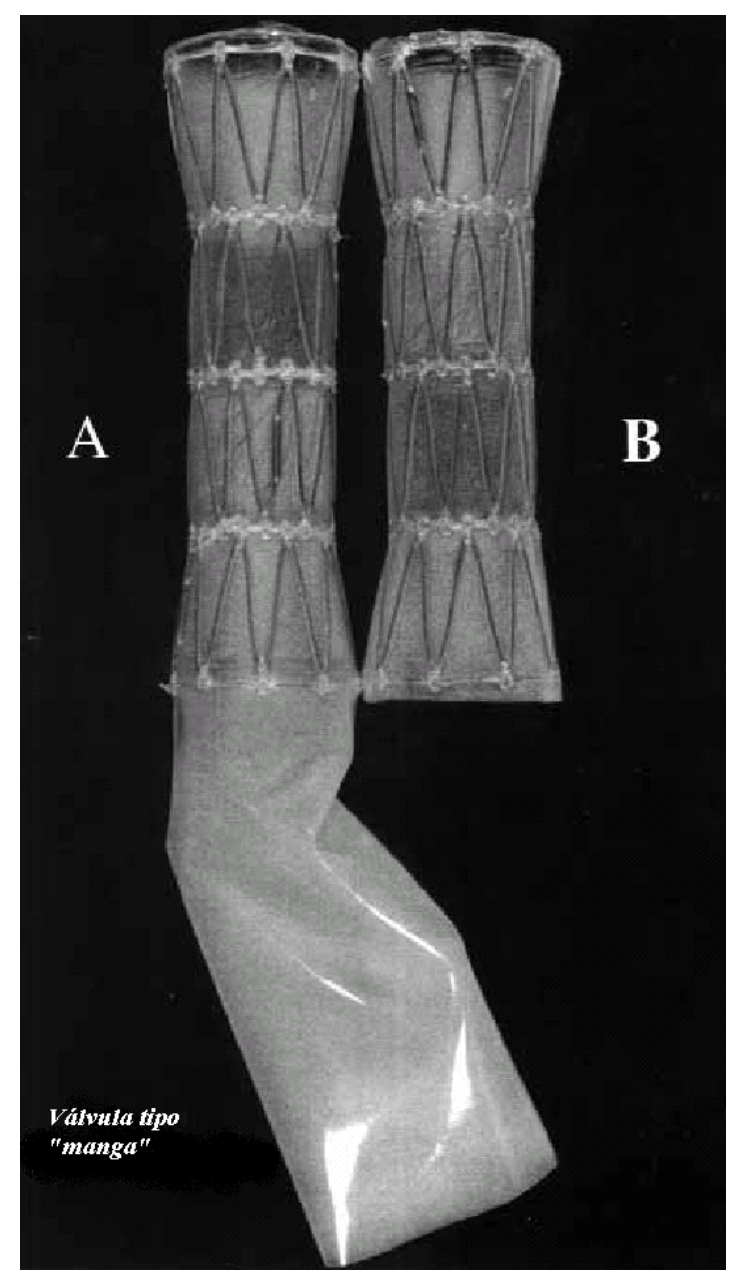

Figura 4- Modelos Z-Stent com (A) e sem(B) válvula anti-refluxo

2001)(Figura 4). Esta PMAE com válvula anti-refluxo (VAR), foi desenvolvida a partir da seguinte metodologia, em estudos in vitro: a propriedade anti-refluxo foi estudada pela colocação da prótese em um cilindro com água a $37^{\circ} \mathrm{C}$, submerginda-a com sua válvula anti-refluxo para baixo. A pressão em centímetros de água necessária para inverter a válvula para dentro da prótese foi medida para várias espessuras de membranas (limites de espessuras examinadas: 0.11 a $0.24 \mathrm{~mm}$ ). Após encaixar a prótese em um tubo oco, a prótese inteira com sua válvula foi mergulhada em água por um minuto permitindo que o revestimento da mesma e a válvula atingissem $37^{\circ} \mathrm{C}$. A prótese foi então retirada e depois novamente submersa verticalmente em água até que a válvula invertesse para dentro da prótese. A pressão em centímetros d'água necessária para inverter a válvula foi 
determinada medindo a distância do nível da água e a extremidade inferior da parte metálica da prótese (Figura 5). Essa foi a pressão que se ultrapassada, potencialmente irá causar refluxo gastroesofágico. Uma vez invertida, a prótese foi retirada e $50 \mathrm{ml}$ de água foram passados através dela para verificar se a válvula retornava à posição original. Para cada espessura de membrana, os testes foram repetidos cinco vezes.

Os resultados demonstraram que, a pressão necessária para inverter a válvula quando a prótese era imersa em água, aumentava de acordo com a espessura da membrana da válvula. Para válvula com membrana de $0.17 \mathrm{~mm}$, a pressão foi de $48 \mathrm{~cm}$ de água a $37^{\circ} \mathrm{C}, \pm 0.4 \mathrm{~cm}$ de erro padrão e essa espessura de membrana de válvula foi usada em todos os pacientes. A pressão não foi afetada pelo comprimento de prótese usada. Para todas as espessuras, a válvula foi apropriadamente revertida com $50 \mathrm{ml}$ de água passados por dentro da prótese.

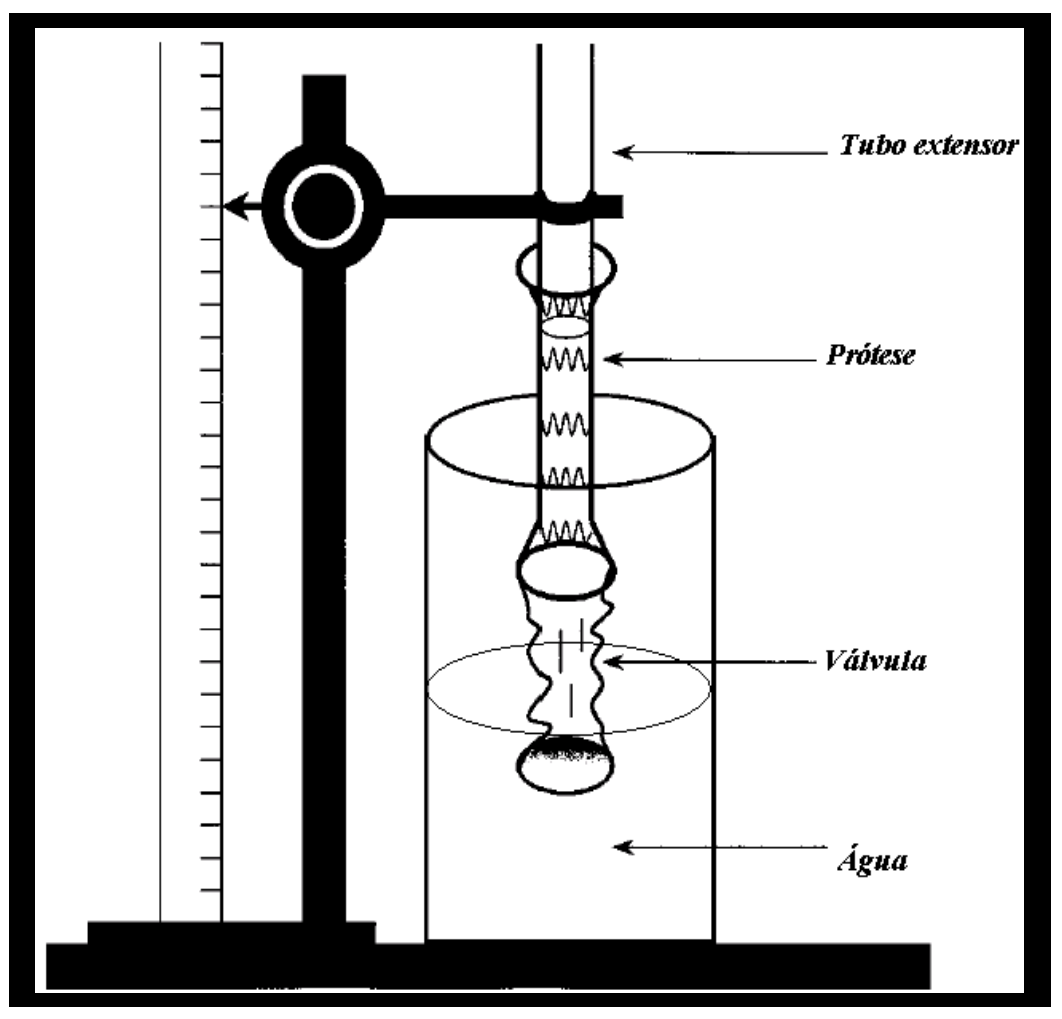

Figura 5- Modelo de teste da válvula anti-refluxo 
Dua et al. (2001) realizou o estudo da PMAE modificada em cães, utilizando próteses Z-Stent padrão e modificadas, com cada cão atuando como seu próprio controle. Cinco cães (2 machos, peso 14, $18 \mathrm{~kg}$; 3 fêmeas, peso 14, 14, $16 \mathrm{~kg}$ ) foram submetidos à esofagostomia com o estoma localizado na base do pescoço. Antes do estudo, todos foram submetidos à endoscopia digestiva alta via esofagostomia sob sedação (Hidrocloreto de tilotamina e hidrocloreto de zolazepan: Telazol $10 \mathrm{mg} / \mathrm{kg}$ ), para documentar a anatomia normal, localizar a posição da JEG e posicionar o fio guia. Foram utilizadas próteses com VAR (EZS-25, $8 \mathrm{~cm}$ comprimento e $18 \mathrm{~mm}$ de diâmetro) e sem VAR (EZS-25, $8 \mathrm{~cm}$ comprimento e $18 \mathrm{~mm}$ de diâmetro) e a inserção através da JEG foi realizada pelas técnicas descritas pelo fabricante. Após a retirada do dispositivo de colocação e o fio guia, o cão foi reexaminado endoscopicamente pela esofagostomia para confirmar se a prótese estava bem posicionada. Enquanto a posição da prótese era monitorizada pela endoscopia, um fio preso à extremidade superior foi suturado à pele próximo à esofagostomia, para prevenir migração da mesma.

$\mathrm{O} \mathrm{pH}$ esofágico de $24 \mathrm{hs}$ foi monitorizado ambulatorialmente usando dois sítios de eletrodos de antimônio e digitrapper (Digitrapper MK III e Probes pH, Medtronic Functional Diagnostics, Shoreview, Minn). Antes do estudo, os eletrodos foram calibrados para pH 1 e pH 7, posicionados a 5 e $10 \mathrm{~cm}$ proximal à JEG e para prevenir migração, o fio foi suturado à pele próximo à esofagostomia. A monitorização de dois sítios de $\mathrm{pH}$ foi utilizado para diferenciar entre evento de refluxo e deglutição. Durante o RGE a mudança de $\mathrm{pH}$ atingiria o sítio distal de gravação de $\mathrm{pH}$ antes do sítio proximal, e durante a deglutição a mudança ocorreria em ordem inversa.

Os animais foram transferidos à unidade de cuidados intensivos por uma hora e após recuperação, enviados às suas jaulas. Vestimentas apropriadas foram utilizadas 
para evitar que eles puxassem ou danificassem o equipamento. Durante cada período de gravação, $1185 \mathrm{ml}$ de dieta líquida (1250 calorias) foi colocada na jaula, e ao final do período de gravação a quantidade de dieta consumida pelos cães foi anotada. A prótese e os eletrodos foram avaliados endoscopicamente para confirmar se não haviam se deslocado, e em seguida foram removidos através da esofagostomia. A análise dos dados foi realizada pelo software Gastrograph (EsopHogram Synetics pH Program, Gastrograph Inc., Shoreview, Minn.). Para cada período de gravação, os seguintes dados foram obtidos: (1) duração do período de gravação, (2) quantidade de comida consumida pelo cão, (3) número de episódios de refluxo, (4) número de episódios de refluxo superiores a cinco minutos, e (5) a fração (\%) do total do período de gravação em que o pH permaneceu abaixo de 4, isto é, o tempo de exposição ácida no esôfago.

Inicialmente dois cães foram estudados usando a prótese sem VAR, no dia seguinte substituída pela prótese com VAR, e três deles foram estudados na ordem inversa.

Os resultados demonstraram que a duração média da monitorização ambulatorial de $\mathrm{pH}$ e o volume de líquido consumido pelos animais foram similares quando utilizadas a prótese com e sem VAR. Houve significativamente mais episódios de refluxos $(\mathrm{pH}<4)$, cada um com duração maior que cinco minutos, usando-se a prótese sem VAR quando comparada com a valvulada. A percentagem de tempo de gravação total em que o $\mathrm{pH}$ do esôfago distal permaneceu abaixo de 4 (tempo de exposição ácida do esôfago) foi de $49 \%$ utilizando-se a prótese sem VAR, comparado com $1 \%(p=0.027)$ quando utilizado a prótese com VAR. Estes resultados permitiram concluir que a prótese valvulada foi eficaz em aliviar a disfagia, comparável à sem VAR, assim como em prevenir o refluxo gastroesofágico. 
Em 1999, Dua et al. utilizaram pela primeira vez esse tipo de PMAE com VAR em 11 pacientes com carcinoma de esôfago distal e da JEG. O primeiro paciente tratado apresentou queixas de RGE, atribuído ao fato de a espessura da membrana da válvula de $0.11 \mathrm{~mm}$ inverter facilmente (com $22 \pm 0.2 \mathrm{~cm}$ de água a $37^{\circ} \mathrm{C}$ ). Uma segunda prótese com espessura da membrana da válvula de $0.17 \mathrm{~mm}$, mais resistente à inversão $(48 \pm 0.4$ cm de água a $37^{\circ} \mathrm{C}$ ), foi introduzida, obtendo-se melhora dos sintomas e, a partir daí, foram utilizadas apenas próteses com membrana dessa espessura. Embora esse estudo piloto não tenha incluído grupo controle, dois pacientes apresentaram queixas de RGE após a colocação da prótese sem VAR através da JEG. Seus sintomas melhoraram substancialmente após a colocação da prótese valvulada através da prótese prévia.

No presente estudo, os resultados na paliação da disfagia pela PMAE com VAR foram estatisticamente significativos em todas avaliações, sendo comparados àqueles obtidos com próteses sem VAR (BARTELSMAN et al., 2000; OLSEN et al.,1999; SEGALIN et al., 1997) e com VAR (DO et al., 2001; DUA et al., 2001; OSUGI et al., 2002; Shim et al., 2005). O estudo radiológico contrastado com bário mostrou bom fluxo anterógrado coincidente com a melhora do índice de disfagia. O diâmetro da válvula (25 $\mathrm{mm}$ ) é maior que o diâmetro do corpo da prótese $(18 \mathrm{~mm})$ e por esse motivo, alimentos que passaram facilmente pela prótese, passariam pela válvula, com a mesma facilidade.

A nossa análise mostrou melhora no sintoma de regurgitação após a colocação da prótese no $7^{\circ}, 30^{\circ}$ e $120^{\circ}$ dias, $p=0.001, p=0.008$ e $p=0.039$, respectivamente. Durante 0 seguimento, seis pacientes apresentaram sintomas de regurgitação; cinco $(75 \%)$ deles apresentaram ruptura da válvula ao exame endoscópico, sendo estatisticamente significativo $(p=0,005)$. Essa intercorrência foi observada num período variável de 34 a 237 dias, e os pacientes foram orientados a seguirem medidas comportamentais para 
prevenção de RGE. Nenhum apresentou pneumonia aspirativa como descrito em estudos utilizando PMAE sem VAR (BARTELSMAN et al., 2000; WESTON \& SHARMA, 1999).

O uso de IBP foi prescrito para uma paciente que apresentou gastrite hemorrágica e nos seis casos nos quais se verificou ruptura da válvula.

A válvula é capaz de inverter para dentro da prótese sob gradiente de pressão aumentada, como eructação, tosse e vômitos, possibilitando o RGE. Essa propriedade permite propositadamente que o paciente possa vomitar. Dua et al. (2001) descreveram em sua casuística, um paciente com obstrução do intestino delgado secundária à doença metastática e, apesar da válvula anti-refluxo, foi capaz de vomitar. Outro paciente, submetido a quimioterapia após a colocação da prótese, desenvolveu anorexia, e foi imprescindível a realização de gastrostomia percutânea para alimentação. Embora a luz da prótese, pelo exame endoscópico, estivesse pérvia, o bário injetado no estômago via gastrostomia não refluiu para o esôfago, portanto não houve necessidade de transformar a gastrostomia em jejunostomia.

Após a inserção da prótese, observou-se aumento do sintoma de dor retroesternal em aproximadamente metade dos pacientes, sendo esta persistente e significativa nas avaliações do $7^{\circ}, 30^{\circ}$ e $120^{\circ}$ dias $(p=0.011, p=0,025$ e $p=0,020)$. O sintoma de dor relatado pelos pacientes em outros estudos (DO et al., 2001; DUA et al., 2001 e SHIM et al., 2005) com utilização de próteses nessa mesma localização, foi de menor intensidade e transitória.

O parâmetro nutricional avaliado objetivamente pelo índice de massa corpórea, não apresentou alteração significativa após o tratamento. Entretanto, deve-se ressaltar que, previamente à inserção da prótese, os pacientes apresentavam perda ponderal 
progressiva nos últimos meses, e após a mesma, houve estabilização temporária desse parâmetro.

Pacientes que necessitam de tratamento paliativo devido à estenose esofágica por doença maligna, apresentam comprometimento marcante na qualidade de vida, particularmente aqueles com disfagia absoluta. Alguns deles são extremamente debilitados, dependentes de cuidados de enfermagem, necessitam de nutrição parenteral ou por sondas de alimentação, e apresentam curto período de ingestão oral após a inserção de próteses, independente das mesmas estarem permeáveis.

Entre as várias características que auxiliam determinar quais os pacientes que se beneficiarão com o tratamento por próteses, tais como idade, extensão do tumor, localização da estenose e estadiamento TNM, o único fator preditivo relevante foi o índice de Karnofsky (YAJIMA et al., 2004).

Dua et al. (2001), utilizando a mesma prótese deste estudo, descreveram crescimento tumoral envolvendo os dois terços proximais do estômago, entretanto não foi possível avaliar se esse fato influenciou no funcionamento da válvula, devido ao paciente encontrar-se em extrema caquexia e parou de se alimentar.

No presente estudo, não se verificou crescimento tumoral nas extremidades da prótese, porém a hiperplasia tecidual benigna nas extremidades da mesma ocorreu em quatro pacientes, tendo surgido após três a nove meses, confirmada pela histologia. $\mathrm{O}$ tratamento da hiperplasia por coagulação tecidual com plasma de argônio foi realizado em dois pacientes; em outro, foi passada uma segunda prótese com VAR e, no último, procedeu-se à passagem de sonda enteral, em função de intensa caquexia e anorexia.

O maior índice de complicações, como sangramento, migração da prótese e perfuração correlacionou-se com a localização topográfica da lesão. Não houve relação 
direta entre a maior incidência de complicações em pacientes submetidos a tratamento com RT e QT.

A rica rede vascular da região, com ramos da artéria gástrica esquerda e a posição da prótese na JEG com força de expansão radial exercida de forma assimétrica, com conseqüente ulceração de uma das superfícies do tumor, podem justificar o sangramento observado. Bartelsman et al. (2000) relataram risco de hemorragia 1.27 vezes maior nas lesões de esôfago distal quando comparado ao esôfago proximal.

O fato de a localização topográfica não apresentar área de sustentação distal para a prótese, ficando a sua porção distal livre na câmara gástrica, a torna mais susceptível ao deslocamento e à migração. A ocorrência de migração como resultado da modificação não é esperada, pois a válvula repousará predominantemente na região subcárdica e fúndica do estômago, distante das contrações do antro. As próteses colocadas em terço distal e/ou cárdia têm 1.4 vezes maior risco de migrar, em comparação com as de terço proximal (BARTELSMAN et al., 2000). Utilizando o mesmo tipo de prótese, Dua et al. (2001) descreveram $18 \%$ de migração, e no presente estudo houve quatro $(13,30 \%)$ migrações, que ocorreram após dois dias e dois, quatro e 24 meses posteriormente à introdução da prótese. Em um dos pacientes, a lesão neoplásica não era circunferencial e de consistência amolecida, o que predispõe à migração. No entanto, nos demais, as lesões eram circunferenciais e nenhum deles realizou RT ou QT.

A angulação existente na inserção do esôfago no estômago está relacionada à maior dificuldade na transposição de lesões tumorais nessa localização; tornando-as tecnicamente mais difíceis de serem dilatadas para o posicionamento do sistema introdutor e liberar a prótese, com maior risco de perfuração. No nosso estudo, essa 
complicação ocorreu em $10 \%$ dos pacientes e foi correlacionada com $100 \%$ de mortalidade.

Embora a pHmetria esofágica de $24 \mathrm{~h}$ seja considerada como o método padrão ouro para avaliar a ocorrência do refluxo gastroesofágico, esse procedimento não foi efetuado, pois levou-se em conta a recusa formal do paciente em realizá-lo, por ser método invasivo e não trazer qualquer benefício para quem apresenta expectativa de vida de cerca de 4 meses. Todavia, esse método já foi realizado em cães, na elaboração da prótese, e demonstrou qual a espessura ideal da membrana valvular para evitar o refluxo gastroesofágico (DUA KS, 2001), e que o tempo de exposição ácida esofágica foi significativamente menor, quando se utilizou prótese modificada comparada com a padrão.

O motivo mais provável para alguns pacientes terem apresentado sintomas diurnos de refluxo é que a válvula inverte-se para dentro da prótese em gradientes de alta pressão, por exemplo, durante episódios de tosse e espirro, que podem induzir a refluxo de "estresse". A válvula anti-refluxo colaba e se torna "pinçada" com a elevação da pressão intragástrica. Contudo, se a pressão intragástrica sobe excessivamente, por exemplo, durante náuseas ou vômitos (acima de $48 \mathrm{~cm}$ de água a $37^{\circ} \mathrm{C}$ para uma válvula de membrana com $0,17 \mathrm{~mm}$ de espessura), a válvula inverte para dentro do corpo da prótese. No desenho do modelo, essa propriedade foi intencional para permitir ao paciente eructar ou vomitar. Para evitar que a válvula permanecesse nessa posição, recomendou-se aos pacientes que, após qualquer episódio de aumento da pressão intraabdominal, no surgimento de sintomas de refluxo gastroesofágico e antes de deitar, ingerissem um copo com água, com o intuito de reverter a válvula. 
Laasch et al (2002), realizaram estudo comparativo da prótese com e sem válvula. Foram estudados dois grupos de 25 pacientes cada, com disfagia por neoplasia avançada de esôfago distal e/ou cárdia, com o objetivo de avaliar sintomas de refluxo. No grupo tratado com prótese sem válvula, 24 (96\%) apresentaram sintomas de refluxo, necessitando de tratamento com inibidores de bomba de prótons; no grupo com válvula, três (4\%) pacientes apresentaram sintomas de refluxo, um deles necessitando de terapia. Um dos pacientes do grupo com prótese sem válvula apresentou aspiração brônquica como complicação, e faleceu de pneumonia aspirativa. Apesar de esses três pacientes apresentarem sintomas de refluxo, não foi descrito nenhum exame comprobatório de ruptura ou incompetência da válvula. Em nosso estudo, foi constatado em um paciente com pirose, a ocorrência de refluxo através da válvula íntegra, notando-se um desvio de eixo na posição da prótese em relação ao tumor. Esse fato alerta para a possibilidade do posicionamento da prótese interferir no funcionamento da válvula.

A durabilidade do poliuretano em relação ao ácido do suco gástrico e à bile é desconhecida. A válvula pode desintegrar-se com o tempo. Isso ocorreu com um de nossos pacientes que se submeteu à endoscopia após cerca de 10 semanas de colocação da prótese. Há planos de substituição da constituição do revestimento de poliuretano das próteses por materiais de maior durabilidade, que resistiriam mais a bile e a ácido. Apesar da necessidade dessa discussão junto a representantes da Wilson-Cook Medical, Inc., Winston-Salem, NC, a durabilidade das próteses a longo prazo, não deve ser a preocupação principal na paliação de pacientes com doença terminal. Atualmente, as próteses em questão não são recomendadas para obstruções benignas do esôfago ou para doença do refluxo gastroesofágico (DUA KS, 2001). 
Existem outros modelos de PMAE com mecanismo anti-refluxo; tal qual a prótese FerX-Ella (Ella-CS, Hradec Kralove, República Tcheca), que apresenta desenho semelhante à Esophageal Z-Stent W/Dua Anti-reflux Valve. Homs et al. (2004), comparando a FerX-Ella com e sem VAR, mostraram que a prótese com VAR, apesar de obter sucesso na paliação da disfagia, não foi capaz de prevenir o refluxo, confirmado por pHmetria de 24h. Características físicas divergentes podem justificar essa discrepância em relação aos resultados obtidos por Dua et al. (2001). A válvula da FerX-Ella é constituída de polietileno, o seu comprimento é de $47 \mathrm{~mm}$ e o folheto de polietileno tem espessura de $0.15 \mathrm{~mm}$, enquanto que a DUA Z-Stent utiliza o poliuretano, material que é mais firme e se deforma menos, seu comprimento é de $80 \mathrm{~mm}$, quase o dobro da FerXElla, e a espessura do folheto é de $0.17 \mathrm{~mm}$. A prótese FerX-Ella apresentou elevado índice de migração, de aproximadamente $25 \%$ e, apesar de ser totalmente coberta, o que a torna menos fixa à lesão, esse resultado coincide com os demonstrados no nosso estudo $(13,30 \%)$, sugerindo que a região do cárdia é local propício à ocorrência de migração.

Shim et al. (2005) publicaram estudo comparativo de três diferentes modelos de próteses utilizadas em tumores da junção esofagogástrica. Uma do tipo convencional, sem mecanismo anti-refluxo e duas com mecanismos valvulares anti-refluxo, uma tipo tricúspide (Dostent M.I. Tech. Co., Inc., Pyongtack, Korea) e outra do tipo longa de folhetos em S (M.I. Tech. Co., Inc., Pyongtack, Korea), ambas localizadas internamente na prótese. A Z-Stent DUA AR, por sua vez, possui a válvula estendida externamente, após a extremidade distal. Foram 36 pacientes, randomizados em grupos de 12, e a ocorrência de refluxo foi verificada em todos os pacientes por meio de pHmetria $24 \mathrm{~h}$. O grupo com a prótese com válvula longa tipo S obteve o melhor resultado na prevenção 
dos sintomas de RGE e no resultado da pHmetria 24h, e foi estatisticamente superior aos outros dois $(p<0.005$ e $p<0.001$ respectivamente). Um dos pacientes com prótese com válvula anti-refluxo tipo tricúspide desenvolveu pneumonia aspirativa. Não houve diferença estatística na mortalidade em 30 dias ou na média de sobrevida.

O desenvolvimento de próteses com mecanismo anti-refluxo, que foram desenhadas especificamente para atender às peculiaridades da região da junção esofagogástrica, ainda necessita aperfeiçoamento e mais estudos controlados. 


\section{Conclusões}

\section{Conclusões}

O emprego da prótese metálica auto-expansível com válvula anti-refluxo em pacientes portadores de tumores avançados da junção esofagogástrica permitiu concluir que:

- Foi eficaz na paliação da disfagia.

- Impediu a ocorrência de manifestações broncoaspirativas.

- Não interferiu na evolução do parâmetro nutricional.

- Não influiu na avaliação da qualidade de vida. 



\section{Referências bibliográficas}

Referências bibliográficas

Adefna Perez RI, Leal Mursulí A, Izquierdo Lara FT, Castellanos González J.

Transthoracic esophagectomy vs transhiatal esophagectomy for the surgical treatment of esophageal carcinoma. (Protocol for a Cochrane Review). In: The Cochrane Library, Issue 1, 2004. Oxford: Update Software.

Alexandrou A, Davis PA, Law S, et al. Squamous cell carcinoma and adenocarcinoma of the lower third of the esophagus and gastric cardia: similarities and differences. Dis Esophagus 2002; 15: 290-295.

Allum WH, Griffin SM, Watson A, et al. Guidelines for the management of oesophageal and gastric cancer. Gut 2002; 50(Suppl V):v1-v23.

Altorki N, Kent M, Ferrara C, Port J. Three-field lymph node dissection for squamous cell and adenocarcinoma of the esophagus. Ann Surg 2002; 236(2): 177-183.

Aoki T, Osaka Y, Takagi Y, et al. Comparative study of self-expandable metallic stent and bypass surgery for inoperable esophageal cancer. Dis Esophagus 2001; 14: 208211. 
Baron TH, Morgan DE, Yates MR. Use of expandable metal stents for malignant gastrointestinal luminar obstruction. Clin Perspectives Gastroenterol. 1999; Jan/Feb 16-24.

Bartelsman JFW, Bruno MJ, Jensema AJ, et al. Palliation of patients with esophagogastric neoplasms by insertion of a covered expandable modified GianturcoZ endoprosthesis: experiences in 153 patients. Gastrointest Endosc 2000; 51: 134-8.

Blot W J, Devesa S S, Kneller R W, Fraumeni J F, Jr. Rising incidence of adenocarcinoma of the esophagus and gastric cardia. JAMA 1991; 265: 1287-1289.

Cameron AJ. Epidemiology of Barrett's esophagus and adenocarcinoma. Dis Esophagus. 2002; 15(2): 106-108.

Cantero R, Torres AJ, Hernando F, et al. Palliative treatment of esophageal cancer: self-expanding metal stents versus Postlethwait technique. Hepato-Gastroenterol 1999; 46(26):971-6.

Chang JT, Katzka DA. Gastroesophageal reflux disease, Barrett esophagus, and esophageal adenocarcinoma. Arch Intern Med 2004;164:1482-1488.

Corley DA, Buffler PA. Oesophageal and gastrica adenocarcinomas: analysis of regional variations using the Cancer Incidence in five continents database. Int $\mathrm{J}$ Epidemiol 2001; 30: 1415-1425.

de Boer AGEM, van Lanschot JJB, van Sandick JW, et al. Quality of life after transhiatal compared with extended transthoracic resection for adenocarcinoma of the esophagus. J Clin Oncol 2004; 22: 4202-4208.

Do YS, Choo SW, Suh SW, et al. Malignant esophagogastrica junction obstruction: palliative treatment with an antireflux valve stent. J Vasc Interv Radiol 2001; 12: 647651.

Dua KS, Kozarek R, Kim J. Self-expanding metal esophageal stent with anti-reflux mechanism. Gastrointest Endosc. 2001; 53(6): 603-12.

Ellis HF, Gibb PS, Watkins E. Esophagogastrectomy- A safe, widely applicable, and expeditious form of palliation for patients with carcinoma of the esophagus and cardia. Ann Surg 1983; 198(4) oct: 531-39. 
El-Serag HB, Mason AC, Petersen N, Key CR. Epidemiological differences between adenocarcinoma of the oesophagus and adenocarcinoma of the gastric cardia in the USA. Gut 2002; 50: 368-372.

Enzinger PC, Mayer RJ. Medical progress: esophageal cancer. N Eng J Med 2003; 349(23): 2241-2252.

Ferrari AP, Libera Jr ED, Morais M, et al. Brazilian expandable metal stent in palliative treatment of esophagus tumor: report of the first case. GED 1997; 16(1): 31-34.

Gore R M. Esophageal cancer- clinical and pathological features. Radiol Clin North America. 1997; 35(2) mar: 243-63.

Guang-sen L, Hui-nan Z, Zhi-fan H. Long-term results of surgical treatment of esophageal and cardia carcinoma. Chinese Med J. 1986; 99(9): 747-748.

Harbord M, Dawes RFH, Barr H, et al. Palliation of patients with dysphagia due to advanced esophageal cancer by endoscopic injection of cisplatin/epinephrine injectable gel. Gastrointest Endosc 2002; 56: 644-51.

Homs MYV, Wahab PJ, Siersema PD, et al. Esophageal stents with antireflux valve for tumors of the distal esophagus and gastric cardia: a randomized trial. Gastrointest Endosc 2004;60:695-702.

Inoue H. Treatment of esophageal and gastric tumors. Endoscopy 2001; 33(2): 119125.

Jacobson BC, Hirota W, Baron TH, Leighton JA, Faigel DO. The role of endoscopy in the assessment and treatment of esophageal cancer. Gastroint Endosc 2003; 57(7): 817-822.

Johansson J, DeMeester T, Hagen JA, et al. En bloc vs transhiatal esophagectomy for stage T3 N1 Adenocarcinoma of the distal esophagus. Arch Surg 2004; 139(6): 627633.

Knyrim K, Wagner H, Bethge N. A controlled trial of an expansible metal stent for palliation of esophageal obstruction due to inoperable cancer. N Eng J Med. 1993 329(18) oct: 1302-1307.

Köcher M, Dlouhy M, Neoral C, et al. Esophageal stent with antireflux valve for tumor involving the cardia: Work in progress. J Vasc Interv Radiol 1998; 9:1007-1010. 
Kubba AK, Krasner N. An update in the palliative management of malignant dysphagia. Eur J Surg Oncol 2000; 26:116-129.

Laasch H, Martin DF, Do YS, Köcher M, Valette PJ. Interventional Radiology for the management of inoperable carcinoma of the oesophagus. Endoscopy 2003; 35(12): 1049-1058.

Laasch HU, Marriott A, Wilbraham L, et al. Effectiveness of open versus antireflux stents for palliation of distal esophageal carcinoma and prevention of gastroesophageal reflux. Radiol 2002; 225: 359-365.

Lagergreen J, Bergströn R, Nyrén O. Association between body mass index and adenocarcinoma of the esophagus and gastrica cardia. Ann Intern Med 1999; 130: 883-890.

Law S, Wong J. Therapeutic options for esophageal cancer. J Gastroenterol Hepatol. 2004; 19:4-12.

Lee $\mathrm{SH}$. The role of oesophageal stenting in the non-surgical management of oesophageal strictures. Br J Radiol 2001; 74: 891-900.

Leiper K, Morris AI, Treatment of oesophago-gastric tumours. Endoscopy 2002; 34: 139-145.

Lerut T, Coosemans W, Decker G, et al. Extracapsular lymph node involvement is a negative prognostic factor in T3 adenocarcinoma of the distal esophagus and gastroesophageal junction. J Thorac Cardiovasc Surg 2003;126:1121-8.

Liu JF, Wang QZ, Hou J. Surgical treatment for cancer of the oesophagus and gastric cardia in Hebei, China. Br J Surg 2004; 91: 90-98.

Manzoni G, Pedrazzani Z, Verlato G, et al. Comparison of old and new TNM systems for nodal staging in adenocarcinoma of the gastro-oesophageal junction. Br J Surg 2004; 91: 296-303.

Mariette C, Balon JM, Maunoury V, et al. Value of endoscopic ultrasonography as a predictor of long-term survival in oesophageal carcinoma. Br J Surg 2003; 90: 13671372. 
Mattioli S, Di Simone MP, Ferruzi L, et al. Surgical therapy for adenocarcinoma of the cardia: modalities of recurrence and extension of resection. Dis Esophagus 2001; 14: 104-109.

May A, Selmaier M, Hochberger J, et al. Memory metal stents for palliation of malignant obstruction of the oesophagus and cardia. Gut 1995; 37: 309-13.

McManus K, Khan I, McGuigan J. Self-expanding oesophageal stents: strategies for reintervention. Endoscopy 2001; 33(7): 601-604.

Mizumoto Y, Matsuda K, Itoh Y et al. Trial use of a Gore-Tex covered Ultraflex stent with reflux preventive action for cardioesophageal cancer [abstract]. Gastrointest Endosc 1997; 45: AB35.

Monga SPS, Wadleigh $\mathrm{R}$, Sharma A, et al. Intratumoral therapy of cisplatin/epinephrine injectable gel for palliation in patients with obstructive esophageal cancer. Am J Clin Oncol 2000; 23(4): 386-392.

Moreto M. Diagnosis of esophagogastric tumors. Endoscopy 2005; 37(1): 26-32.

Mosca F, Consoli A, Stracqualursi A, et al. Comparative retrospective study on the use of plastic prostheses and self-expanding metal stents in the palliative treatment of malignant strictures of the esophagus and cardia. Dis Esophagus 2003; 16: 119-125.

Moura EGH. Tratamento paliativo do câncer do esôfago por endoprótese e tubo gástrico isoperistáltico. Tese de doutorado da Faculdade de Medicina da Universidade de São Paulo 2000.

Nakamura T, Ide $\mathrm{H}$, Eguchi R. Adenocarcinoma of the esophagogastric junction: a summary of responses to a questionnaire on adenocarcinoma of the esophagus and esophagogastric junction in Japan. Dis Esophagus 2002; 15: 219-25.

Noguchi T, Wada S, Takeno S, et al. Two-step three-field lymph node dissection is beneficial for thoracic esophageal carcinoma. Dis Esophagus 2004; 17: 27-31.

Nunes CC, Waechter FL, Sampaio JA et al. Comparative post-operative study of prostheses, with and without an anti-reflux valve system, in the palliative treatment of esophageal carcinoma. Hepatogastroenterology 1999; 46: 2859-2864. 
Olsen E, Thyregaard R, Kill J. Esophacoil expanding stent in the management of patients with nonresectable malignant esophageal or cardiac neoplasm: a prospective study. Endoscopy 1999; 31(6): 417-420.

Osugi $\mathrm{H}$, Lee $\mathrm{S}$, Higashino $\mathrm{M}$ et al. Usefullness of self-expandable metallic stent with na antireflux mechanism as a palliation for malignant strictures at the gastroesophageal junction. Surg Endoscopy 2002; 16(10): 1478-82.

Parkin DM, Pisani P, Ferlay J. Estimates of the worldwide incidence of 25 major cancers in 1990. Int J Cancer 1999; 80:827-841.

Penman ID, Shen EF. EUS in advanced esophageal cancer. Gastroint Endosc 2002, 56(4): S2-S6.

Pinotti HW, Felix VN, Raia A. Revisão e análise crítica das técnicas de restabelecimento do trânsito esofágico. An paul med cir 1978; 105(4): 01-28.

Richel DJ, Vervenne WL. Systemic treatment of oesophageal cancer. Eur J Gastroenterol Hepatol 2004; 16: 249-254.

Sallum RA. Adenocarcinoma da cárdia: resultados do tratamento cirúrgico fundamentado na topografia tumoral. Tese de doutorado da Faculdade de Medicina da Universidade de São Paulo 1996.

Sandha GS, Marcon NE. Expandable metal stents for benign esophageal obstruction. Gastrosintest Endosc Clin N Am 1999;9:437-46.

Segalin A, Bonavina L, Carazzone A, et al. Improving results of esophageal stenting: a study on 160 consecutive unselected patients. Endoscopy 1997; 29: 701-709.

Shim CS, Jung IS, Cheon YK, et al. Self-Expanding Metal Stent with Antireflux Mechanism. Endoscopy 2005; 37: 335 \pm 339 .

Siersema PD, Hop WCJ, Dees J, et al. Coated self-expanding metal stents versus latex prothesis for esophagogastric cancer with special reference to prior radiation and chemotherapy: a controlled, prospective study. Gastrointest Endosc 1998; 47: 113-20.

Siersema PD, Marcon N, Vakil N. Metal stents for tumors of the distal esophagus and gastric cardia. Endoscopy 2003; 35(1):79-85. 
Siewert JR, Feith M, Werner M. Adenocarcinoma of the esophagogastric junction. Ann Surg 2000; 232(3): 353-361.

Siewert JR, Holscher AH, Becker K, Gossner W. Kardiakarzinom:Versuch einer therapeutisch relevanten Klassifikation. Chirurgie 1987; 58:25-34.

Song HY, Do YS, Han YM et al. Covered, expandable esophageal metallic stent tubes: experiences in 119 patients. Radiology 1994; 193:689 \pm 695.

Spechler SJ. The Natural History of Dysplasia and Cancer in Esophagitis and Barrett Esophagus. J Clin Gastroenterol 2003;36(Suppl. 1):S2-S5.

Spencer GM, Thorpe SM, Blackman GM, et al. Laser augmented by brachytherapy versus laser alone in the palliation of adenocarcinoma of the oesophagus and cardia: a randomised study. Gut 2002; 50(2): 224-227.

Tachibana M, Kinugasa S, Yoshimura H, et al. Extended esophagectomy with 3-field lymph node dissection for esophageal cancer. Arch Surg 2003; 138: 1383-1389.

Tytgat GNJ, Bartelink H, Bernards $\mathrm{R}$ et al. Câncer of the esophagus and gastric cárdia: recent advances. Dis Esophagus 2004; 17(1): 10-26.

Udagawa $\mathrm{H}$, Akiyama $\mathrm{H}$. Surgical treatment of esophageal cancer: Tokyo experience of the three-field technique. Dis Esophag 2001; 14: 110-114.

Valbuena J. Palliation of gastroesophageal carcinoma with endoscopic insertion of a new antireflux prosthesis. Gastrointestinal Endosc 1984; 30: 241-243.

Viscaino AP, Moreno V, Lambert R, Parkin DM. Time trend incidence of both major histologic types of esophageal carcinomas in selected countries, 1973-1995. Int J Cancer 2002; 99(6): 860-80.

Walter C, Zilling T, Perfekt R, Moller T. Strongly increasing incidence of adenocarcinoma of the esophagus and gastric cardia. Lakartidningen. 2004; 101(3):180-3. 
Wang KK. Detection and staging of esophageal cancers. Curr Op Gastroenterol 2004; 20: 381-385.

Wei JT, Shaheen N. The changing epidemiology of esophageal adenocarcinoma. Sem Gastrointest Dis. 2003; 14(3):112-27.

Weston AP, Sharma P. Early and late complications from esophageal metallic stents. Am J Gastroenterol 1999; 94: 2602.

Wijnhoven BPL, Siersema PD, Hop WCJ, et al. Adenocarcinomas of the distal oesophagus and gastric cardia are one clinical entity. Br J Surg 1999; 86: 529-535.

Yajima K, Kanda T, Nakagawa S, et al. Self-expandable metallic stents for palliation of malignant esophageal obstruction: special reference to quality of life and survival of patients. Dis Esophagus 2004; 17: 71-75. 